\title{
Structures and Dimensions of Vector Valued Jacobi Forms of Degree Two
}

\author{
by
}

Tomoyoshi IBUKIYAma

\begin{abstract}
We give a complete characterization of vector valued holomorphic Jacobi forms of degree two of index one in the sense of Ziegler by the Taylor expansion and vector valued Siegel modular forms of various weights. By this characterization, we also give explicit dimension formulas for spaces of vector valued holomorphic Jacobi forms of index one of degree two, using those for vector valued Siegel modular forms and a certain surjectivity theorem on the Witt operator (the restriction operator to the diagonals). Our characterization also gives a concrete way to give the plus subspace of the space of Siegel modular forms of half-integral weight.
\end{abstract}

2010 Mathematics Subject Classification: Primary 11F50; Secondary 11F46, 11F37.

Keywords: Jacobi forms, Siegel modular forms, dimension formula, half-integral weight.

\section{$\S 1$. Introduction}

We consider the space $J_{(k, j), 1}\left(\Gamma_{2}^{J}\right)$ of holomorphic Jacobi forms of degree 2 of index 1 of weight $\operatorname{det}^{k} \operatorname{Sym}_{j}$, where $\mathrm{Sym}_{j}$ is the symmetric tensor representation of GL(2) of degree $j$. The Jacobi forms are holomorphic functions $f(\tau, z)$ on $\mathfrak{H}_{2} \times \mathbb{C}^{2}$ which satisfy certain automorphy under the Jacobi modular group $\Gamma_{2}^{J}$ (of level one), where $\mathfrak{H}_{2}$ is the Siegel upper half-space of degree two. To characterize this space, we use the Taylor expansion of $f(\tau, z)$ along $z=0$. A Jacobi form $f$ of index one is an even function with respect to $z$ and when $f$ is of degree two, we can show that it is determined by the Taylor coefficients up to degree 2 . We denote by $A_{k, j}\left(\Gamma_{2}\right)$ the space of Siegel modular forms of weight $\operatorname{det}^{k} \mathrm{Sym}_{j}$ of the Siegel modular group $\Gamma_{2}$. Roughly speaking, we obtain a linear mapping from Taylor coefficients of $f(\tau, z)$ up to degree two into the space $A_{k, j}\left(\Gamma_{2}\right) \times A_{k, j+2}\left(\Gamma_{2}\right) \times A_{k+1, j}\left(\Gamma_{2}\right) \times A_{k, j-2}\left(\Gamma_{2}\right)$, and we can show that the space $J_{(k, j), 1}\left(\Gamma_{2}^{J}\right)$ is isomorphic to the image of this mapping.

Communicated by S. Mochizuki. Received August 1, 2014. Revised March 10, 2015.

T. Ibukiyama: Department of Mathematics, Graduate School of Science, Osaka University, Machikaneyama 1-1, Toyonaka, Osaka, 560-0043 Japan;

e-mail: ibukiyam@math.sci.osaka-u.ac.jp

(C) 2015 Research Institute for Mathematical Sciences, Kyoto University. All rights reserved. 
Belonging to this image is described as the condition that some part vanishes under the restriction to the diagonal of $\tau \in \mathfrak{H}_{2}$, which leads to explicit dimension formulas when $k$ is not too small. R. Tsushima gave conjectural dimension formulas for spaces of Jacobi forms of degree two in [20] including more general cases, and in the above case, our formula completely coincides with his conjecture. This formula also gives the dimensions of the plus subspaces of Siegel modular forms of halfintegral weight of degree two of good parity, since the spaces of holomorphic Jacobi forms are isomorphic to such spaces, with character or without character according to the parity of $k$. The results of this paper were used in [12]. Some part of this paper can be generalized to higher degrees without much difficulty, but we would like to treat them on a different occasion.

The paper is organized as follows. After reviewing the definitions and easy properties of Jacobi forms and Siegel modular forms in Section 2, we construct a mapping from the Taylor coefficients of holomorphic Jacobi forms to a sum of vector valued Siegel modular forms in Section 3 (Theorem 3.5). This is similar to the results in [3] and [10], but much more complicated since the relation to the theory of differential operators on Siegel modular forms is not clear and there are no ready-made differential operators as in [8] that we can use to construct the mapping in this case. In Section 4 (Theorem 4.1), we give a characterization of the image of this mapping by using the Witt operator (that is, restriction to the diagonals of $\mathfrak{H}_{2}$ ). In Section 5, by using Theorem 4.1, we first give formulas for the dimensions of spaces of vector valued Jacobi forms in terms of those of vector valued Siegel modular forms and elliptic modular forms for $k \geq 8$ (Theorem 5.3) and then give explicit generating functions of the dimensions (Theorem 5.6). We explain the relation to Siegel modular forms of half-integral weight and also give remarks on small weights. Finally we give numerical tables of the dimensions of spaces of Jacobi forms or Jacobi cusp forms for several small $k$ and $j$.

\section{$\S 2$. Definitions and easy properties}

We denote by $\operatorname{Sp}(n, \mathbb{R})$ the real symplectic group of matrix size $2 n$ :

$$
\operatorname{Sp}(n, \mathbb{R})=\left\{g \in \mathrm{GL}(2 n, \mathbb{R}) ;{ }^{t} g J g=J\right\},
$$

where $J=\left(\begin{array}{cc}0 & -1_{n} \\ 1_{n} & 0\end{array}\right)$ and $1_{n}$ is the $n \times n$ unit matrix. We put $\Gamma_{n}=\operatorname{Sp}(n, \mathbb{R}) \cap$ $M_{2 n}(\mathbb{Z})$. We denote by $\mathfrak{H}_{n}$ the Siegel upper half-space of degree $n$. We fix a (finitedimensional) rational representation $\rho$ of $\operatorname{GL}(n, \mathbb{C})$ and let $V$ be the representation space of $\rho$. For any holomorphic function $F: \mathfrak{H}_{n} \rightarrow V$ and $g=\left(\begin{array}{ll}a & b \\ c & d\end{array}\right) \in \operatorname{Sp}(n, \mathbb{R})$, we write

$$
\left(\left.F\right|_{\rho}[g]\right)(\tau)=\rho(c \tau+d)^{-1} F(g \tau) \quad\left(\tau \in \mathfrak{H}_{n}\right) .
$$


The function $F$ is called a Siegel modular form of weight $\rho$ if $\left.F\right|_{\rho}[\gamma]=F$ for any $\gamma \in \Gamma_{n}$ (with extra boundedness condition at cusps if $n=1$ ). We define the Siegel $\Phi$-operator by

$$
(\Phi F)\left(\tau_{1}\right)=\lim _{t \rightarrow \infty} F\left(\begin{array}{cc}
\tau_{1} & 0 \\
0 & i t
\end{array}\right)
$$

where $\tau_{1} \in \mathfrak{H}_{n-1}$ and $t \in \mathbb{R}, i=\sqrt{-1}$. We say that $F$ is a cusp form if $\Phi(F)=0$. We denote by $A_{\rho}\left(\Gamma_{n}\right)$ and $S_{\rho}\left(\Gamma_{n}\right)$ the spaces of Siegel modular forms and Siegel cusp forms of weight $\rho$, respectively. We denote by $\mathrm{Sym}_{j}$ the symmetric tensor representation of $\operatorname{GL}(n, \mathbb{C})$ of degree $j$. If $\rho=\operatorname{det}^{k} \operatorname{Sym}_{j}$, we write $A_{\rho}\left(\Gamma_{n}\right)=$ $A_{k, j}\left(\Gamma_{n}\right)$ and $S_{\rho}\left(\Gamma_{n}\right)=S_{k, j}\left(\Gamma_{n}\right)$. We note that when $n=2$, we have $A_{k, j}\left(\Gamma_{2}\right)=0$ if $j$ is odd and $A_{k, j}\left(\Gamma_{2}\right)=S_{k, j}\left(\Gamma_{2}\right)$ if $k$ is odd.

Next, we identify $\operatorname{Sp}(n, \mathbb{R})$ as a subgroup of $\operatorname{Sp}(n+1, \mathbb{R})$ by mapping

$$
g=\left(\begin{array}{ll}
a & b \\
c & d
\end{array}\right) \in \operatorname{Sp}(n, \mathbb{R}) \quad \text { to } \quad\left(\begin{array}{llll}
a & 0 & b & 0 \\
0 & 1 & 0 & 0 \\
c & 0 & d & 0 \\
0 & 0 & 0 & 1
\end{array}\right) \in \operatorname{Sp}(n+1, \mathbb{R})
$$

For $\lambda, \mu \in \mathbb{R}^{n}$ and $\kappa \in \mathbb{R}$, we define an element $[(\lambda, \mu), \kappa] \in \operatorname{Sp}(n+1, \mathbb{R})$ by

$$
[(\lambda, \mu), \kappa]=\left(\begin{array}{cccc}
1_{n} & 0 & 0 & { }^{t} \mu \\
\lambda & 1 & \mu & \kappa \\
0 & 0 & 1_{n} & -{ }^{t} \lambda \\
0 & 0 & 0 & 1
\end{array}\right)
$$

The elements $[(\lambda, \mu), \kappa]$ form a subgroup $\mathcal{H}_{n}(\mathbb{R})$ of $\operatorname{Sp}(n+1, \mathbb{R})$, called the Heisenberg group. For any integer $n \geq 1$, we define the real Jacobi group $G_{n}^{J}(\mathbb{R})$ as the subgroup of $\operatorname{Sp}(n+1, \mathbb{R})$ defined by

$$
G^{J}(\mathbb{R})=\left\{g \cdot[(\lambda, \mu), \kappa] ; g \in \operatorname{Sp}(n, \mathbb{R}), \lambda, \mu \in \mathbb{R}^{n}, \kappa \in \mathbb{R}\right\} .
$$

This is a semidirect product of $\operatorname{Sp}(n, \mathbb{R})$ and $\mathcal{H}_{n}(\mathbb{R})$. Again, let $(\rho, V)$ be an irreducible rational representation of $\operatorname{GL}(n, \mathbb{C})$. For any $V$-valued function $f(\tau, z)$ : $\mathfrak{H}_{n} \times \mathbb{C}^{n} \rightarrow V$, any $g=\left(\begin{array}{ll}a & b \\ c & d\end{array}\right) \in \operatorname{Sp}(n, \mathbb{R}) \subset G^{J}(\mathbb{R})$, any $[(\lambda, \mu), \kappa] \in \mathcal{H}_{n}(\mathbb{R})$, and any fixed integer $m \geq 1$, we write

$$
\begin{aligned}
\left.f\right|_{\rho, m}[g] & =e^{m}\left(-z(c \tau+d)^{-1} c^{t} z\right) \rho(c \tau+d)^{-1} f\left(g \tau, z(c \tau+d)^{-1}\right), \\
\left.f\right|_{m} \mid[(\lambda, \mu), \kappa] & =e^{m}\left(\lambda \tau^{t} \lambda+2 \lambda^{t} z+\mu^{t} \lambda+\kappa\right) f(\tau, z+\lambda \tau+\mu),
\end{aligned}
$$

where we write $e^{m}(x)=e(m x)$ and $e(x)=\exp (2 \pi i x)$ for any $x$. This gives an action of $G^{J}(\mathbb{R})$. We write $\mathcal{H}_{n}(\mathbb{Z})=\mathcal{H}_{n}(\mathbb{R}) \cap M_{2 n+2}(\mathbb{Z})$. We set $\Gamma_{n}^{J}=G^{J}(\mathbb{R}) \cap$ $M_{2 n+2}(\mathbb{Z})=\Gamma_{n} \cdot \mathcal{H}_{n}(\mathbb{Z}) \subset G(\mathbb{R})^{J}$ and call it the Jacobi modular group of degree $n$. 
When $n \geq 2$, we say that a $V$-valued holomorphic function $f(\tau, z): \mathfrak{H}_{n} \times \mathbb{C}^{n} \rightarrow V$ is a Jacobi form of weight $\rho$, index $m$ and degree $n$ if

$$
\begin{aligned}
\left.f\right|_{\rho, m}[\gamma] & =f & & \text { for all } \gamma \in \Gamma_{n} \subset \Gamma_{n}^{J}, \\
\left.f\right|_{m}[(\lambda, \mu), \kappa] & =f & & \text { for all }[(\lambda, \mu), \kappa] \in \mathcal{H}_{n}(\mathbb{Z}) .
\end{aligned}
$$

By this condition of automorphy, we have the Fourier expansion of the form

$$
f(\tau, z)=\sum_{(N, r) \in L_{n}^{*} \times \mathbb{Z}^{n}} C(N, r) e(\operatorname{tr}(N \tau)) e\left(r^{t} z\right)
$$

where $L_{n}^{*}$ is the set of $n \times n$ half-integral symmetric matrices. When $n=1$, for the definition of Jacobi forms, we need an extra condition that $C(N, r)=0$ unless $4 m N-{ }^{t} r r$ is positive semidefinite, which is satisfied automatically if $n \geq 2$ (see Ziegler [22]). We say that $f$ is a Jacobi cusp form if $C(N, r)=0$ unless $4 m N-{ }^{t} r r$ is positive definite. We denote by $J_{\rho, m}\left(\Gamma_{n}^{J}\right)$ and $J_{\rho, m}^{\text {cusp }}\left(\Gamma_{n}^{J}\right)$ the space of Jacobi forms and Jacobi cusp forms respectively. When $\rho=\operatorname{det}^{k} \mathrm{Sym}_{j}$, we write $J_{\rho, m}=J_{(k, j), m}$. When $n=2$, we identify the representation space of $\mathrm{Sym}_{j}$ with the space $V_{j}=\mathbb{C}\left[u_{1}, u_{2}\right]_{j}$ of homogeneous polynomials $P\left(u_{1}, u_{2}\right)$ in $u_{1}$ and $u_{2}$ of degree $j$ and realize $\operatorname{Sym}_{j}$ by $P\left(u_{1}, u_{2}\right) \mapsto P\left(\left(u_{1}, u_{2}\right) A\right)$ for $A \in \operatorname{GL}(2, \mathbb{C})$.

In order to characterize a Jacobi cusp form of index one, we introduce the Jacobi-Siegel $\Phi$-operator $\Phi^{J}$ as in [2] as follows. For any $f \in J_{\rho, m}\left(\Gamma_{n}^{J}\right)$ we define

$$
\left(\Phi^{J} f\right)\left(\tau_{1}, z_{1}\right)=\lim _{t \rightarrow \infty} f\left(\left(\begin{array}{cc}
\tau_{1} & 0 \\
0 & i t
\end{array}\right),\left(z_{1}, 0\right)\right),
$$

where $\tau_{1} \in \mathfrak{H}_{n-1}$ and $z_{1} \in \mathbb{C}^{n-1}$.

We give several easy properties when the index $m$ is 1 .

Proposition 2.1. Assume that a Jacobi form $f \in J_{\rho, 1}\left(\Gamma_{n}^{J}\right)$ is of index $m=1$. Then we have the following properties:

(a) The Fourier coefficient $C(N, r)$ of $f$ depends only on $4 N-{ }^{t} r r$. In particular, $f$ is an even function with respect to $z$, that is, $f(\tau,-z)=f(\tau, z)$.

(b) If $\rho\left(-1_{n}\right)=-1 \cdot \mathrm{id}_{V}$, then $J_{\rho, 1}\left(\Gamma_{n}^{J}\right)=0$. In particular, if $n=2$ and $\rho=$ $\operatorname{det}^{k} \operatorname{Sym}_{j}$ and $j$ is odd, then $J_{\rho, 1}\left(\Gamma_{2}^{J}\right)=J_{(k, j), 1}\left(\Gamma_{2}^{J}\right)=0$.

(c) A Jacobi form $f \in J_{\rho, 1}\left(\Gamma_{n}^{J}\right)$ is a cusp form if and only if $\Phi^{J}(f)=0$.

(d) When $n=2$ and $\rho=\operatorname{det}^{k} \mathrm{Sym}_{j}$, regarding $f$ as a homogeneous polynomial in $u=\left(u_{1}, u_{2}\right)$ with holomorphic coefficients, we have $\Phi^{J}(f)=\phi\left(\tau_{1}, z_{1}\right) u_{1}^{j}$ with a Jacobi form $\phi\left(\tau_{1}, z_{1}\right)$ of weight $k+j$ of index one and degree one. When $j>0, \phi\left(\tau_{1}, z_{1}\right)$ is a Jacobi cusp form. 
Proof. Although (a) is well known, we give the proof for completeness. If $4 N_{1}-$ ${ }^{t} r_{1} r_{1}=4 N_{2}-{ }^{t} r_{2} r_{2}$ for $N_{i} \in L_{n}^{*}$ and $r_{i} \in \mathbb{Z}^{n}$, then comparing the diagonal components, we see that the squares of the $i$-th components of $r_{1}$ and $r_{2}$ are congruent modulo 4 for each $i$, so $r_{1} \equiv r_{2} \bmod 2$. So we write $r_{2}=r_{1}+2 \lambda$ with $\lambda \in \mathbb{Z}$ and then $N_{1}=N_{2}-{ }^{t} \lambda \lambda-\left({ }^{t} r_{1} \lambda+{ }^{t} \lambda r_{1}\right) / 2$. We apply the condition of automorphy (2.2) for this $\lambda$. We have

$\sum_{N, r} C(N, r) e(\operatorname{tr}(N \tau)) e\left(-\lambda \tau^{t} \lambda-2 \lambda^{t} z\right) e\left(r^{t} z\right)=\sum_{N, r} C(N, r) e(\operatorname{tr}(N \tau)) e\left(r^{t}(z+\lambda \tau)\right)$

Here by the relation between $N_{1}$ and $N_{2}$ above, we easily see that

$$
e\left(\operatorname{tr}\left(N_{2}-{ }^{t} \lambda \lambda\right) \tau\right) e\left(\left(r_{2}-2 \lambda\right){ }^{t} z\right)=e\left(\operatorname{tr}\left(N_{1} \tau\right)\right) e\left(r_{1}{ }^{t}(z+\lambda \tau)\right)
$$

So we have $C\left(N_{1}, r_{1}\right)=C\left(N_{2}, r_{2}\right)$. By (a), we often write the Fourier coefficients $C(N, r)$ as $C\left(4 N-{ }^{t} r r\right)$. By the action of $-1_{2 n}$, we see that $f(\tau,-z)=$ $\rho\left(-1_{n}\right) f(\tau, z)$ for $f \in J_{\rho, 1}\left(\Gamma_{n}^{J}\right)$, but since $f$ is even in $z$ by (a), we have $J_{\rho, 1}\left(\Gamma_{n}^{J}\right)=0$ if $\rho\left(-1_{n}\right)=-1 \cdot \mathrm{id}_{V}$. This proves (b).

We now prove (c) and (d). Since $f$ is of index one, we write the Fourier expansion as

$$
f(\tau, z)=\sum_{N, r \in L_{n}^{*} \times \mathbb{Z}^{n}} C\left(4 N-{ }^{t} r r\right) e(\operatorname{tr}(N \tau)) e\left({ }^{t} r z\right) .
$$

We write

$$
N=\left(\begin{array}{cc}
N_{1} & n_{0} / 2 \\
{ }^{t} n_{0} / 2 & n_{2}
\end{array}\right)
$$

and $r=\left(r_{1}, r_{2}\right)$, where $N_{1} \in L_{n-1}^{*}$ and $r_{1} \in \mathbb{Z}^{n-1}$. We assume that $4 N-{ }^{t} r r$ is positive semidefinite from now on. Then $n_{2} \geq 0$, and if $n_{2}=0$, then $r_{2}=0$, $r=\left(r_{1}, 0\right)$ and $n_{0}=0$. So we have

$$
4 N-{ }^{t} r r=\left(\begin{array}{cc}
4 N_{1}-{ }^{t} r_{1} r_{1} & 0 \\
0 & 0
\end{array}\right)
$$

in this case. The terms such that $n_{2}>0$ obviously disappear under $\Phi^{J}$ by definition. If we assume that $n_{2}=0$, then $4 N-{ }^{t} r r$ is not positive definite, and if we assume that $f$ is a cusp form, then $C(N, r)=0$. Hence $\Phi^{J}(f)=0$. Conversely, if $\Phi^{J}(f)=0$, then $C(N, r)=C\left(4 N-{ }^{t} r r\right)=0$ if $n_{2}=0$. Now we must show that $C(N, r)=0$ always when $\operatorname{det}\left(4 N-{ }^{t} r r\right)=0$ even if $n_{0} \neq 0$. By automorphy $(2.1)$ for $\left(\begin{array}{cc}U & 0 \\ 0 & { }^{t} U^{-1}\end{array}\right) \in \Gamma_{n}$ with $U \in \operatorname{GL}(n, \mathbb{Z})$, we have $f\left(U \tau^{t} U, z^{t} U\right)=\rho\left({ }^{t} U\right) f(\tau, z)$. This means that

$$
C\left(4 N-{ }^{t} r r\right)=\rho\left({ }^{t} U\right) C\left({ }^{t} U\left(4 N-{ }^{t} r r\right) U\right)
$$


Now if $\operatorname{det}\left(4 N-{ }^{t} r r\right)=0$ (i.e. if $4 N-{ }^{t} r r$ is positive semidefinite but not positive definite), then we have a non-zero vector $v \in \mathbb{Z}^{n}$ such that $\left(4 N-{ }^{t} r r\right) v=0$ and we may assume that the greatest common divisor of the components of $v$ is 1 . Then there exists an element $U \in \mathrm{SL}(n, \mathbb{Z})$ whose $n$-th column is $v$. For this $U$, the last column of ${ }^{t} U\left(4 N-{ }^{t} r r\right) U$ is also zero. This means that the $(n, n)$ component of ${ }^{t} U\left(4 N-{ }^{t} r r\right) U$ is zero. So $C\left({ }^{t} U\left(4 N-{ }^{t} r r\right) U\right)=0$ and hence $C\left(4 N-{ }^{t} r r\right)=0$ for all $N$ and $r$ such that $\operatorname{det}\left(4 N-{ }^{t} r r\right)=0$. By definition, this means that $f$ is a cusp form. This proves (c).

Now we prove (d). For $f \in J_{(k, j), 1}\left(\Gamma_{2}^{J}\right)$ and $\tau=\left(\tau_{i j}\right) \in \mathfrak{H}_{2}, z=\left(z_{1}, z_{2}\right) \in \mathbb{C}^{2}$, we have

$$
\Phi^{J}(f)\left(\tau_{11}, z_{1}\right)=\sum_{\substack{n, r \in \mathbb{Z} \\
4 n-r^{2} \geq 0}} C\left(\begin{array}{cc}
4 n-r^{2} & 0 \\
0 & 0
\end{array}\right) e\left(n \tau_{11}\right) e\left(r z_{1}\right) .
$$

In (2.3), we put $U=\left(\begin{array}{ll}1 & 0 \\ x & 1\end{array}\right)$ with $x \in \mathbb{Z}$. Then

$$
{ }^{t} U\left(\begin{array}{cc}
4 n-r^{2} & 0 \\
0 & 0
\end{array}\right) U=\left(\begin{array}{cc}
4 n-r^{2} & 0 \\
0 & 0
\end{array}\right)
$$

hence

$$
\operatorname{Sym}_{j}\left(\begin{array}{ll}
1 & x \\
0 & 1
\end{array}\right) C\left(\begin{array}{cc}
4 n-r^{2} & 0 \\
0 & 0
\end{array}\right)=C\left(\begin{array}{cc}
4 n-r^{2} & 0 \\
0 & 0
\end{array}\right) .
$$

So, if we write $C\left(\begin{array}{rr}4 n-r^{2} & 0 \\ 0 & 0\end{array}\right)=\sum_{i=0}^{j} c_{i} u_{1}^{j-i} u_{2}^{i} \in \mathbb{C}\left[u_{1}, u_{2}\right]_{j}$, then

$$
\sum_{i=0}^{j} c_{i} u_{1}^{j-i} u_{2}^{i}=\sum_{i=0}^{j} c_{i} u_{1}^{j-i}\left(u_{1} x+u_{2}\right)^{i}
$$

for any $x \in \mathbb{Z}$. Comparing the coefficients of $u_{1}^{j-l} u_{2}^{l}$ for $l \neq j$, we see that $\sum_{i=l}^{j}\left(\begin{array}{l}i \\ l\end{array}\right) c_{i} x^{i-l}=c_{l}$. So taking $x \neq 0$, we see inductively that $c_{i}=0$ if $i \geq 1$. Hence we can write $\Phi^{J}(f)=\phi\left(\tau_{11}, z_{1}\right) u_{1}^{j}$. It is clear that $\phi \in J_{k+j, 1}\left(\Gamma_{1}^{J}\right)$ since $\Phi^{J}(f)$ inherits the automorphic property of $f$. If we write

$$
\phi\left(\tau_{11}, z_{1}\right)=\sum_{n, r} c_{\phi}\left(4 n-r^{2}\right) e\left(n \tau_{11}\right) e\left(r z_{1}\right),
$$

then this is a cusp form if and only if $c_{\phi}(0)=0$, since it is of index one (see [3]). If we denote by $O_{2}$ the $2 \times 2$ zero matrix and apply (2.3) for $O_{2}$ and $U=\left(\begin{array}{ll}1 & x \\ 0 & 1\end{array}\right)$ with $0 \neq x \in \mathbb{Z}$, then since ${ }^{t} U O_{2} U=O_{2}$, we see that in the polynomial $C\left(O_{2}\right)$ in $u=\left(u_{1}, u_{2}\right)$, all the coefficients except that of $u_{2}^{j}$ should vanish. Since $c_{\phi}(0)$ is the coefficient of $u_{1}^{j}$ in $C\left(O_{2}\right)$, we have $c_{\phi}(0)=0$ if $j>0$. Hence $\phi\left(\tau_{11}, z\right)$ is a cusp form if $j>0$. 
Next we explain the theta expansion restricting to the case when $n=2$ and $m=1$. For any $\nu \in(\mathbb{Z} / 2 \mathbb{Z})^{2}$, we define

$$
\vartheta_{\nu}(\tau, z)=\sum_{p \in \mathbb{Z}^{2}} e\left(\left(p+\frac{\nu}{2}\right) \tau^{t}\left(p+\frac{\nu}{2}\right)+2\left(p+\frac{\nu}{2}\right) t_{z}\right) .
$$

Then it is well known that for each $f \in J_{(k, j), 1}\left(\Gamma_{2}^{J}\right)$ there exist $V_{j}$-valued holomorphic functions $c_{i j}(\tau)(0 \leq i, j \leq 1)$ uniquely determined by $f$ such that

$$
\begin{aligned}
& f(\tau, z)= \\
& c_{00}(\tau) \vartheta_{00}(\tau, z)+c_{01}(\tau) \vartheta_{01}(\tau, z)+c_{10}(\tau) \vartheta_{10}(\tau, z)+c_{11}(\tau) \vartheta_{11}(\tau, z) .
\end{aligned}
$$

We call this the theta expansion. On the other hand, write the Taylor expansion of $f$ along $z=0$ as

$$
f(\tau, z)=f_{0}(\tau)+f_{20}(\tau) z_{1}^{2}+f_{11}(\tau) z_{1} z_{2}+f_{02}(\tau) z_{2}^{2}+O\left(z^{4}\right) .
$$

Here $O\left(z^{4}\right)$ means a series of $z_{1}, z_{2}$ such that the lowest total degree is not less than 4 . We note that the Taylor coefficients $f_{0}(\tau)$ etc. are $V_{j}$-valued, so we often write $f_{0}(\tau)=f_{0}(\tau, u)$ and so on when we emphasize that $f_{0}$ is a polynomial in $u=\left(u_{1}, u_{2}\right)$ with holomorphic coefficients.

Lemma 2.2. The linear mapping from $f \in J_{(k, j), 1}\left(\Gamma_{2}^{J}\right)$ to $\left(f_{0}, f_{20}, f_{11}, f_{02}\right)$ (the Taylor coefficients of $f$ of degree up to two) is injective.

Proof. We prove this by showing that the coefficients $c_{i j}(\tau)$ of the theta expansion (2.4) are uniquely determined by these Taylor coefficients. We set $\vartheta_{\nu}(\tau)=\vartheta_{\nu}(\tau, 0)$. We write $\partial_{i j}=\frac{1}{2 \pi i} \frac{\partial}{\partial \tau_{i j}}$. Then we have the following well known relation:

$$
\left.\frac{1}{(2 \pi i)^{2}} \frac{\partial^{2} \vartheta_{\nu}}{\partial z_{i} \partial z_{j}}\right|_{z=0}=2\left(1+\delta_{i j}\right) \partial_{i j} \vartheta_{\nu}(\tau)
$$

Hence if we write

$$
\Theta(\tau)=\left(\begin{array}{cccc}
\vartheta_{00}(\tau) & \vartheta_{01}(\tau) & \vartheta_{10}(\tau) & \vartheta_{11}(\tau) \\
\partial_{11} \vartheta_{00}(\tau) & \partial_{11} \vartheta_{01}(\tau) & \partial_{11} \vartheta_{10}(\tau) & \partial_{11} \vartheta_{11}(\tau) \\
\partial_{12} \vartheta_{00}(\tau) & \partial_{12} \vartheta_{01}(\tau) & \partial_{12} \vartheta_{10}(\tau) & \partial_{12} \vartheta_{11}(\tau) \\
\partial_{22} \vartheta_{00}(\tau) & \partial_{22} \vartheta_{01}(\tau) & \partial_{22} \vartheta_{10}(\tau) & \partial_{22} \vartheta_{11}(\tau)
\end{array}\right)
$$

then using the theta expansion and the Taylor expansion, we obtain

$$
\Theta(\tau)\left(\begin{array}{l}
c_{00}(\tau) \\
c_{01}(\tau) \\
c_{10}(\tau) \\
c_{11}(\tau)
\end{array}\right)=\left(\begin{array}{c}
f_{0}(\tau) \\
\frac{1}{2(2 \pi i)^{2}} f_{20}(\tau) \\
\frac{1}{2(2 \pi i)^{2}} f_{11}(\tau) \\
\frac{1}{2(2 \pi i)^{2}} f_{02}(\tau)
\end{array}\right)
$$


As was shown in [10, p. 591], $\operatorname{det}(\Theta(\tau))$ is the non-zero cusp form $\chi_{5}$ of weight 5 of $\Gamma_{2}$ with sign character $\operatorname{sgn}: \operatorname{Sp}\left(2, \mathbb{F}_{2}\right) \cong S_{6} \rightarrow S_{6} / A_{6} \cong\{ \pm 1\}$ where $S_{6}$ and $A_{6}$ are the symmetric and alternating groups on six letters. So the solution of the linear equation (2.6) is unique.

Our next task is to describe the image of this mapping. We will do this in the following sections.

\section{$\S 3$. A mapping to Siegel modular forms}

We construct a certain mapping from Jacobi forms to Siegel modular forms using the Taylor coefficients. In this section, we take the index $m \geq 1$ arbitrary. Identifying the representation space of $\operatorname{Sym}_{j}$ with $V_{j}=\mathbb{C}\left[u_{1}, u_{2}\right]_{j}$, we write $f(\tau, z) \in$ $J_{(k, j), m}\left(\Gamma_{2}^{J}\right)$ as $f(\tau, u, z)$ to emphasize that this is a polynomial in $u$. Then the condition (2.1) of automorphy with respect to $\Gamma_{2}$ can be written as

$$
\begin{aligned}
f\left(\gamma \tau, u(c \tau+d)^{-1}, z(c \tau\right. & \left.+d)^{-1}\right) \\
& =\operatorname{det}(c \tau+d)^{k} e^{m}\left(z(c \tau+d)^{-1} c^{t} z\right) f(\tau, u, z)
\end{aligned}
$$

for any $\gamma=\left(\begin{array}{ll}a & b \\ c & d\end{array}\right) \in \Gamma_{2}$. We write the Taylor expansion of $f$ as in the last section. By comparing the constant term of the Taylor expansion of both sides of (3.1), it is clear that $f_{0}=f_{0}(\tau, u) \in A_{k, j}\left(\Gamma_{2}\right)$ and

$$
f_{0}\left(\gamma \tau, u(c \tau+d)^{-1}\right)=\operatorname{det}(c \tau+d)^{k} f_{0}(\tau, u)
$$

We would like to describe the coefficients $f_{20}, f_{11}, f_{02}$ by Siegel modular forms. For the sake of simplicity, we write

$$
f_{2}(\tau, z, u)=\frac{1}{2(2 \pi i)^{2}}\left(f_{20}(\tau, u) z_{1}^{2}+f_{11}(\tau, u) z_{1} z_{2}+f_{02}(\tau, u) z_{2}^{2}\right) .
$$

Then $f_{2}$ can be regarded as a $W_{j, 2}$-valued holomorphic function where

$$
W_{j, 2}=\mathbb{C}\left[u_{1}, u_{2}\right]_{j} \otimes\left(\mathbb{C} z_{1}^{2}+\mathbb{C} z_{1} z_{2}+\mathbb{C} z_{2}^{2}\right),
$$

which is identical to the space of polynomials in $u=\left(u_{1}, u_{2}\right)$ and $z=\left(z_{1}, z_{2}\right)$ of degree $j$ and of degree 2 respectively. We can define a natural action of $\mathrm{GL}(2, \mathbb{C})$ on $W_{j, 2}$ by $P(u, z) \mapsto P(u A, z A)$ for $P \in W_{j, 2}$ and $A \in \operatorname{GL}(2, \mathbb{C})$. We assume that $j$ is even. If $j=0$, the space $W_{0,2}$ is isomorphic to $\mathrm{Sym}_{2}$ and irreducible. When $j \geq 2$, the action of $\mathrm{GL}(2, \mathbb{C})$ on $W_{j, 2}$ is not irreducible, and the irreducible decomposition is given by

$$
W_{j, 2} \cong \operatorname{Sym}_{j+2} \oplus \operatorname{det} \operatorname{Sym}_{j} \oplus \operatorname{det}^{2} \operatorname{Sym}_{j-2} .
$$


Assume for the moment that $f_{0}=0$. Then comparing the degree two terms of the Taylor expansion of (3.1) along $z=0$, it is easy to see that

$$
f_{2}\left(g \tau, u(c \tau+d)^{-1}, z(c \tau+d)^{-1}\right)=\operatorname{det}(c \tau+d)^{k} f_{2}(\tau, u, z) .
$$

This means that $f_{2}$ can be regarded as an element of $A_{k, j+2}\left(\Gamma_{2}\right) \oplus A_{k+1, j}\left(\Gamma_{2}\right) \oplus$ $A_{k+2, j-2}\left(\Gamma_{2}\right)$ if $f_{0}=0$. So it is natural to expect that $f_{2}$ is close to an element of $A_{k, j+2}\left(\Gamma_{2}\right) \oplus A_{k+1, j}\left(\Gamma_{2}\right) \oplus A_{k+2, j-2}\left(\Gamma_{2}\right)$ even when $f_{0} \neq 0$. In fact, if we adjust $f_{2}$ to $\xi_{2}$ by means of some derivatives of $f_{0}$, then we can really prove that $\xi_{2}$ has the desired property. We will see how to do the adjustment below.

First we prepare some notation. For any function $h\left(x_{1}, x_{2}\right)$ of $x=\left(x_{1}, x_{2}\right)$, we define a column vector $\operatorname{grad}_{x}(h)$ by

$$
\operatorname{grad}_{x}(h)={ }^{t}\left(\frac{\partial h}{\partial x_{1}}, \frac{\partial h}{\partial x_{2}}\right) .
$$

We write $u=\left(u_{1}, u_{2}\right)$ and $z=\left(z_{1}, z_{2}\right)$. We fix any $V_{j}$-valued holomorphic function $F(\tau, u)$ and write $F(\tau, u)=\sum_{i=0}^{j} \phi_{i}(\tau) u_{1}^{j-i} u_{2}^{i}$. For this fixed $F$, we write

$$
\begin{aligned}
F_{1}(\tau, u, z) & =z \cdot \operatorname{grad}_{u}(F) \\
& =\sum_{i=0}^{j} \phi_{i}(\tau)\left[(j-i) u_{1}^{j-i-1} u_{2}^{i} z_{1}+i u_{1}^{j-i} u_{2}^{i-1} z_{2}\right], \\
F_{2}(\tau, u, z)= & z \cdot \operatorname{grad}_{u}\left(F_{1}\right) \\
= & \sum_{i=0}^{j} \phi_{i}(\tau)\left[(j-i)(j-i-1) u_{1}^{j-2-i} u_{2}^{i} z_{1}^{2}\right. \\
& \left.\quad+2 i(j-i) u_{1}^{j-1-i} u_{2}^{i-1} z_{1} z_{2}+i(i-1) z u_{1}^{j-i} u_{2}^{i-2} z_{2}^{2}\right],
\end{aligned}
$$

where the dot $\cdot$ is the usual matrix multiplication.

Lemma 3.1. Assume that $F=F(\tau, u) \in A_{k, j}\left(\Gamma_{2}\right)$. Then for $l=1,2$, and for any $\gamma=\left(\begin{array}{ll}a & b \\ c & d\end{array}\right) \in \Gamma_{2}$, we have

$$
F_{l}\left(\gamma \tau, u(c \tau+d)^{-1}, z(c \tau+d)^{-1}\right)=\operatorname{det}(c \tau+d)^{k} F_{l}(\tau, u, z) .
$$

Proof. We have $F(\gamma \tau, u)=\operatorname{det}(c \tau+d)^{k} F(\tau, u(c \tau+d))$ by the assumption. It is obvious that $z \cdot \operatorname{grad}_{u}(F(\gamma \tau, u))=z \cdot\left(\operatorname{grad}_{u} F\right)(\gamma \tau, u)=F_{1}(\gamma \tau, u, z)$. By the chain rule, we can show that

$$
z \cdot \operatorname{grad}_{u}(F(\tau, u(c \tau+d)))=z(c \tau+d)\left(\operatorname{grad}_{u} F\right)(\tau, u(c \tau+d)),
$$

which is equal to $F_{1}(\tau, u(c \tau+d), z(c \tau+d))$ by definition. This proves the assertion for $F_{1}$. Repeating the same calculation for $F_{2}$, we have the result for $F_{2}$. 
For later use, we give some formulas (for general $n$ ) on differential operators. For general $n$, we write the components of $\tau \in \mathfrak{H}_{n}$ as $\tau=\left(\tau_{i j}\right)_{1 \leq i, j \leq n}$. We write

$$
\frac{\partial}{\partial \tau}=\left(\frac{1+\delta_{i j}}{2} \frac{\partial}{\partial \tau_{i j}}\right)_{1 \leq i, j \leq n} \text { and } \frac{\partial f}{\partial \tau}=\left(\frac{1+\delta_{i j}}{2} \frac{\partial f}{\partial \tau_{i j}}\right)_{1 \leq i, j \leq n},
$$

where $f$ is a holomorphic function $f(\tau)$ on $\mathfrak{H}_{n}$. For any complex vectors $x=$ $\left(x_{1}, \ldots, x_{n}\right), y=\left(y_{1}, \ldots, y_{n}\right) \in \mathbb{C}^{n}$ independent of $\tau$, we define the differential operator $\partial_{\tau}[x, y]$ by

$$
\partial_{\tau}[x, y]=x \frac{\partial}{\partial \tau}^{t} y=\sum_{1 \leq i \leq j \leq n} \frac{x_{i} y_{j}+x_{j} y_{i}}{2} \cdot \frac{\partial}{\partial \tau_{i j}} .
$$

Obviously we have $\partial_{\tau}[x, y]=\partial_{\tau}[y, x]$.

Lemma 3.2. For $x, y \in \mathbb{C}^{n}, \tau \in \mathfrak{H}_{n}, g=\left(\begin{array}{ll}a & b \\ c & d\end{array}\right) \in \operatorname{Sp}(n, \mathbb{R})$ and a holomorphic function $f(\tau)$ on $\mathfrak{H}_{n}$, we have

$$
\begin{aligned}
\partial_{\tau}[x, y] \operatorname{det}(c \tau+d) & =\operatorname{det}(c \tau+d) \times x(c \tau+d)^{-1} c^{t} y, \\
\partial_{\tau}[x, y]\left(\operatorname{det}(c \tau+d)^{-k}\right) & =-k \operatorname{det}(c \tau+d)^{-k} x(c \tau+d)^{-1} c^{t} y, \\
\partial_{\tau}[x, y]\left((c \tau+d)^{-1}\right) & =-(c \tau+d)^{-1} c \frac{{ }^{t} x y+{ }^{t} y x}{2}(c \tau+d)^{-1}, \\
\partial_{\tau}[x, y](g \tau) & ={ }^{t}(c \tau+d)^{-1} \frac{{ }^{t} x y+{ }^{t} y x}{2}(c \tau+d)^{-1}, \\
\partial_{\tau}[x, y](f(g \tau)) & =x(c \tau+d)^{-1}\left(\frac{\partial f}{\partial \tau}(g \tau)\right){ }^{t}(c \tau+d)^{-1}{ }^{t} y .
\end{aligned}
$$

We omit the proof, since these can be easily proved by matrix calculations. Now we come back to the case $n=2$. For $k \neq 1$, we set

$$
\begin{aligned}
\alpha_{1} & =\frac{1}{2 \pi i} \frac{\left(2-4 j+j^{2}-4 k+3 j k+2 k^{2}\right) m}{2(k-1)(k+j)(2 k+j-2)}, \\
\alpha_{2} & =\frac{1}{2 \pi i} \frac{-(j+k-2) m}{(k-1)(k+j)(2 k+j-2)}, \\
\alpha_{3} & =\frac{1}{2 \pi i} \frac{m}{2(k-1)(k+j)(2 k+j-2)} .
\end{aligned}
$$

When $k=1$, we set $\alpha_{1}=\alpha_{2}=\alpha_{3}=0$. We fix $f \in J_{(k, j), m}\left(\Gamma_{2}^{J}\right)$ and for the constant term $f_{0}$ of $f$, we set $F(\tau, u)=f_{0}(\tau, u)$. We define

$$
\xi_{2}(\tau, u, z)=f_{2}(\tau, u, z)-\alpha_{1} \partial_{\tau}[z, z] F-\alpha_{2} \partial_{\tau}[u, z] F_{1}-\alpha_{3} \partial_{\tau}[u, u] F_{2} .
$$

Proposition 3.3. Notation being as above, for any $\gamma=\left(\begin{array}{ll}a & b \\ c & d\end{array}\right) \in \Gamma_{2}$, we have

$$
\xi_{2}\left(\gamma \tau, u(c \tau+d)^{-1}, z(c \tau+d)^{-1}\right)=\operatorname{det}(c \tau+d)^{k} \xi_{2}(\tau, u, z)
$$


First we will show the following proposition.

Proposition 3.4. Defining $F_{1}$ and $F_{2}$ for any $F=F(\tau, u) \in A_{k, j}\left(\Gamma_{2}\right)$ as before by (3.2) and (3.3), we have

$$
\begin{array}{r}
\left(\partial_{\tau}[z, z] F\right)\left(g \tau, u(c \tau+d)^{-1}, z(c \tau+d)^{-1}\right)-\operatorname{det}(c \tau+d)^{k}\left(\partial_{\tau}[z, z] F\right)(\tau, u, z) \\
=\operatorname{det}(c \tau+d)^{k}\left[k\left(z(c \tau+d)^{-1} c^{t} z\right) F(\tau, u)+\left(u(c \tau+d)^{-1} c^{t} z\right) F_{1}(\tau, u, z)\right], \\
\left(\partial_{\tau}[z, u] F_{1}\right)\left(g \tau, u(c \tau+d)^{-1}, z(c \tau+d)^{-1}\right)-\operatorname{det}(c \tau+d)^{k}\left(\partial_{\tau}[z, u] F_{1}\right)(\tau, u, z) \\
=\operatorname{det}(c \tau+d)^{k}\left[\frac{j}{2}\left(z(c \tau+d)^{-1} c^{t} z\right) F(\tau, u)\right. \\
\left.+\left(k+\frac{j}{2}\right)\left(z(c \tau+d)^{-1} c^{t} u\right) F_{1}(\tau, u, z)+\frac{1}{2}\left(u(c \tau+d)^{-1} c^{t} u\right) F_{2}(\tau, u, z)\right], \\
\left(\partial_{\tau}[u, u] F_{2}\right)\left(g \tau, u(c \tau+d)^{-1}, z(c \tau+d)^{-1}\right)-\operatorname{det}(c \tau+d)^{k}\left(\partial_{\tau}[u, u] F_{2}\right)(\tau, u, z) \\
=\operatorname{det}(c \tau+d)^{k}\left[2(j-1)\left(z(c \tau+d)^{-1} c^{t} u\right) F_{1}(\tau, u, z)\right. \\
\left.+(k+j-2)\left(u(c \tau+d)^{-1} c^{t} u\right) F_{2}(\tau, u, z)\right] .
\end{array}
$$

Proof. We consider a general function $G(\tau, u, z)$ which is holomorphic in $\tau$ and a polynomial in $u$ and $z$. We assume that

$$
G\left(g \tau, u(c \tau+d)^{-1}, z(c \tau+d)^{-1}\right)=\operatorname{det}(c \tau+d)^{k} G(\tau, u, z) .
$$

We make operators $\partial_{\tau}[x, y]$ for $(x, y)=(z, z),(u, z)$ and $(u, u)$ act on both sides of (3.12). The action on RHS is given by

$$
\begin{aligned}
& \partial_{\tau}[x, y]\left(\operatorname{det}(c \tau+d)^{k}\right) G(\tau, u, z)+\operatorname{det}(c \tau+d)^{k}\left(\partial_{\tau}[x, y] G\right)(\tau, u, z) \\
& \quad=k \operatorname{det}(c \tau+d)^{k}\left(x(c \tau+d)^{-1} c^{t} y\right) G(\tau, u, z)+\operatorname{det}(c \tau+d)^{k}\left(\partial_{\tau}[x, y] G\right)(\tau, u, z) .
\end{aligned}
$$

Next we check the action on LHS. We write $\partial_{\tau}[x, y] G$ as $\left(\partial_{\tau}[x, y] G\right)(\tau, u, z, x, y)$ for a moment since this depends on $x$ and $y$. (Later we will set $x, y$ to be $z$ or $u$ but this does not matter.) By the formula (3.8), the action of $\partial_{\tau}[x, y]$ on the $g \tau$ part of LHS is given by

$$
\left(\partial_{\tau}[x, y] G\right)\left(g \tau, u(c \tau+d)^{-1}, z(c \tau+d)^{-1}, x(c \tau+d)^{-1}, y(c \tau+d)^{-1}\right) .
$$

If we replace $x$ or $y$ by $u$ or $z$, we can write this part for $(x, y)=(z, z),(x, y)=$ $(u, z),(x, y)=(u, u)$ as

$$
\begin{aligned}
& \left(\partial_{\tau}[z, z] G\right)\left(g \tau, u(c \tau+d)^{-1}, z(c \tau+d)^{-1}\right), \\
& \left(\partial_{\tau}[u, z] G\right)\left(g \tau, u(c \tau+d)^{-1}, z(c \tau+d)^{-1}\right), \\
& \left(\partial_{\tau}[u, u] G\right)\left(g \tau, u(c \tau+d)^{-1}, z(c \tau+d)^{-1}\right) .
\end{aligned}
$$


Now we consider the action on the $u$ part and the $z$ part of LHS. By the chain rule, the action on $u(c \tau+d)^{-1}$ is given by

$$
u \partial_{\tau}[x, y]\left((c \tau+d)^{-1}\right)\left(\operatorname{grad}_{u} G\right)\left(g \tau, u(c \tau+d)^{-1}, z(c \tau+d)^{-1}\right) .
$$

By (3.6), this is equal to

$$
-\frac{1}{2} u(c \tau+d)^{-1} c\left({ }^{t} x y+{ }^{t} y x\right)(c \tau+d)^{-1}\left(\operatorname{grad}_{u} G\right)\left(g \tau, u(c \tau+d)^{-1}, z(c \tau+d)^{-1}\right) .
$$

If $(x, y)=(z, z)$, then this is equal to

$$
\begin{aligned}
-\left(u(c \tau+d)^{-1} c^{t} z\right) & \left(z(c \tau+d)^{-1}\right)\left(\operatorname{grad}_{u} G\right)\left(g \tau, u(c \tau+d)^{-1}, z(c \tau+d)^{-1}\right) \\
& =-\left(u(c \tau+d)^{-1} c^{t} z\right)\left(z \cdot \operatorname{grad}_{u} G\right)\left(g \tau, u(c \tau+d)^{-1}, z(c \tau+d)^{-1}\right) .
\end{aligned}
$$

If $(x, y)=(u, z)$, then this is equal to

$$
\begin{aligned}
-\frac{1}{2}\left(u(c \tau+d)^{-1} c^{t} u\right)\left(z(c \tau+d)^{-1}\right)\left(\operatorname{grad}_{u} G\right)\left(g \tau, u(c \tau+d)^{-1}, z(c \tau+d)^{-1}\right) \\
-\frac{1}{2}\left(u(c \tau+d) c^{t} z\right)\left(u(c \tau+d)^{-1}\right)\left(\operatorname{grad}_{u} G\right)\left(g \tau, u(c \tau+d)^{-1}, z(c \tau+d)^{-1}\right) \\
=-\frac{1}{2}\left(u(c \tau+d)^{-1} c^{t} u\right)\left(z \cdot \operatorname{grad}_{u} G\right)\left(g \tau, u(c \tau+d)^{-1}, z(c \tau+d)^{-1}\right) \\
-\frac{1}{2}\left(u(c \tau+d) c^{t} z\right)\left(u \cdot \operatorname{grad}_{u} G\right)\left(\left(g \tau, u(c \tau+d)^{-1}, z(c \tau+d)^{-1}\right) .\right.
\end{aligned}
$$

If $(x, y)=(u, u)$, then this is equal to

$$
\begin{aligned}
-\left(u(c \tau+d)^{-1} c^{t} u\right) & \left(u(c \tau+d)^{-1}\right)\left(\operatorname{grad}_{u} G\right)\left(g \tau, u(c \tau+d)^{-1}, z(c \tau+d)^{-1}\right) \\
& =-\left(u(c \tau+d)^{-1} c^{t} z\right)\left(u \cdot \operatorname{grad}_{u} G\right)\left(g \tau, u(c \tau+d)^{-1}, z(c \tau+d)^{-1}\right) .
\end{aligned}
$$

In the same way, the action on the $z(c \tau+d)^{-1}$ part is given as follows. For $(x, y)=$ $(z, z)$, we have

$$
-\left(z(c \tau+d)^{-1} c^{t} z\right)\left(z \cdot \operatorname{grad}_{z} G\right)\left(g \tau, u(c \tau+d)^{-1}, z(c \tau+d)^{-1}\right) .
$$

For $(x, y)=(u, z)$, we have

$$
\begin{aligned}
-\frac{1}{2}(z(c \tau & \left.+d)^{-1} c^{t} z\right)\left(u \cdot \operatorname{grad}_{z} G\right)\left(g \tau, u(c \tau+d)^{-1}, z(c \tau+d)^{-1}\right) \\
& -\frac{1}{2}\left(z(c \tau+d)^{-1} c^{t} u\right)\left(z \cdot \operatorname{grad}_{z} G\right)\left(g \tau, u(c \tau+d)^{-1}, z(c \tau+d)^{-1}\right) .
\end{aligned}
$$

For $(x, y)=(u, u)$, we have

$$
-\left(z(c \tau+d)^{-1} c^{t} u\right)\left(u \cdot \operatorname{grad}_{z} G\right)\left(g \tau, u(c \tau+d)^{-1}, z(c \tau+d)^{-1}\right) .
$$


Now, by definition, $z \cdot\left(\operatorname{grad}_{u} F\right)=F_{1}$ and $z \cdot\left(\operatorname{grad}_{u} F_{1}\right)=F_{2}$. If $P$ is any homogeneous polynomial of degree $j$ in $u$ and of degree $l$ in $z$, then $u \cdot \operatorname{grad}_{u} P=j P$ and $z \cdot \operatorname{grad}_{z} P=l P$ as is well known. So if $F=f_{0}(\tau, u) \in A_{k, j}\left(\Gamma_{2}\right)$, then for $l=1,2$, we have

$$
u \cdot \operatorname{grad}_{u} F_{l}=(j-l) F_{l}, \quad z \cdot \operatorname{grad}_{z} F_{l}=l F_{l} .
$$

Moreover,

$$
\begin{aligned}
u \cdot \operatorname{grad}_{z}\left(F_{2}\right) & =2 z_{1}\left(u_{1} \frac{\partial^{2} F}{\partial u_{1}^{2}}+u_{2} \frac{\partial^{2} F}{\partial u_{1} \partial u_{2}}\right)+2 z_{2}\left(u_{1} \frac{\partial^{2} F}{\partial u_{1} \partial u_{2}}+u_{2} \frac{\partial^{2} F}{\partial u_{2}^{2}}\right) \\
& =2(j-1)\left(z_{1} \frac{\partial F}{\partial u_{1}}+z_{2} \frac{\partial F}{\partial u_{2}}\right)=2(j-1) F_{1} .
\end{aligned}
$$

By applying the formula for general $G$ to the case $G=F, F_{1}$ and $F_{2}$ and using the above relations and Lemma 3.1, Proposition 3.4 is now obvious.

Proof of Proposition 3.3. First we assume that $k=1$. We see that $A_{1, j}\left(\Gamma_{2}\right)=0$ for any $j \geq 0$ since any Jacobi form of $\operatorname{SL}(2, \mathbb{Z})$ of weight 1 of any index is zero by [17] and so the coefficient of $u_{2}^{j}$, and hence all coefficients of $u_{1}^{j-i} u_{2}^{i}$ in elements of $A_{1, j}\left(\Gamma_{2}\right)$ are zero. Hence we need not adjust at all and $f_{2}$ itself is automorphic.

Now assume that $k \geq 2$. Since $f(\tau, u, z) \in J_{(k, j), m}\left(\Gamma_{2}^{J}\right)$, for any $\gamma=\left(\begin{array}{ll}a & b \\ c & d\end{array}\right) \in \Gamma_{2}$ we have

$$
f\left(\gamma \tau, u(c \tau+d)^{-1}, z(c \tau+d)^{-1}\right)=\operatorname{det}(c \tau+d)^{k} e^{m}\left(z(c \tau+d)^{-1} c^{t} z\right) f(\tau, u, z) .
$$

Comparing the order two terms of the Taylor expansion of both sides along $z=0$, we have

$$
\begin{aligned}
& 2(2 \pi i)^{2} f_{2}\left(\gamma \tau, u(c \tau+d)^{-1}, z(c \tau+d)^{-1}\right) \\
& \quad=(2 \pi i m)\left(z(c \tau+d)^{-1} c^{t} z\right) \operatorname{det}(c \tau+d)^{k} f_{0}(\tau, u)+2(2 \pi i)^{2} \operatorname{det}(c \tau+d)^{k} f_{2}(\tau, u, z) .
\end{aligned}
$$

By Proposition 3.4, for $F=f_{0}$ we see that

$$
\begin{array}{r}
\alpha_{1}\left(\left(\partial_{\tau}[z, z] F\right)\left(\gamma \tau, u(c \tau+d)^{-1}, z(c \tau+d)^{-1}\right)-\operatorname{det}(c \tau+d)^{k}\left(\partial_{\tau}[z, z] F\right)(\tau, u, z)\right) \\
+\alpha_{2}\left(\left(\partial_{\tau}[u, z] F_{1}\right)\left(\gamma \tau, u(c \tau+d)^{-1}, z(c \tau+d)^{-1}\right)-\operatorname{det}(c \tau+d)^{k}\left(\partial_{\tau}[u, z] F_{1}\right)(\tau, u, z)\right) \\
+\alpha_{3}\left(\left(\partial_{\tau}[u, u] F_{2}\right)\left(\gamma \tau, u(c \tau+d)^{-1}, z(c \tau+d)^{-1}\right)-\operatorname{det}(c \tau+d)^{k}\left(\partial_{\tau}[u, u] F_{2}\right)(\tau, u, z)\right) \\
=\left(2(2 \pi i)^{2}\right)^{-1}(2 \pi i m)\left(z(c \tau+d)^{-1} c^{t} z\right) \operatorname{det}(c \tau+d)^{k} F(\tau, u) .
\end{array}
$$

Hence we have the assertion.

Now to write $\xi_{2}(\tau, u, z)$ more concretely using elements of $A_{k, j+2}\left(\Gamma_{2}\right) \oplus$ $A_{k+1, j}\left(\Gamma_{2}\right) \oplus A_{k+2, j-2}\left(\Gamma_{2}\right)$, we give the irreducible decomposition of $V_{j, 2}$ more 
concretely. In order to specify the basis of the decomposition, we note the following fact. Let $P(u, z)$ be a polynomial in $u=\left(u_{1}, u_{2}\right)$ and $z=\left(z_{1}, z_{2}\right)$ and $A=\left(\begin{array}{ll}a_{11} & a_{12} \\ a_{21} & a_{22}\end{array}\right) \in \mathrm{GL}(2, \mathbb{C})$. Set $z \cdot \operatorname{grad}_{u}(P(u, z))=P_{1}(u, z)$. Then we have

$$
\begin{aligned}
z \cdot \operatorname{grad}_{u}[P(u A, z A)]= & z_{1}\left(\frac{\partial P}{\partial u_{1}}(u A, z A) a_{11}+\frac{\partial P}{\partial u_{2}}(u A, z A) a_{12}\right) \\
& +z_{2}\left(\frac{\partial P}{\partial u_{1}}(u A, z A) a_{21}+\frac{\partial P}{\partial u_{2}}(u A, z A) a_{22}\right) \\
= & \left(a_{11} z_{1}+a_{21} z_{2}\right) \frac{\partial P}{\partial u_{1}}(u A, z A)+\left(a_{12} z_{1}+a_{22} z_{2}\right) \frac{\partial P}{\partial u_{2}}(u A, z A) \\
= & P_{1}(u A, z A) .
\end{aligned}
$$

So assume that $P^{(i)}(u, z)$ is a basis over $\mathbb{C}$ of some representation space of $\operatorname{GL}(2, \mathbb{C})$ by the action defined by $P^{(i)}(u A, z A)$. Then the space spanned by $z \cdot \operatorname{grad}_{u}\left(P^{(i)}\right)$ is also invariant and the representation matrix of $A$ with respect to $P^{(i)}$ is the same as the one with respect to $P_{1}^{(i)}$. For example, for the basis $u_{1}^{j-i} u_{2}^{i}(0 \leq i \leq j)$ of the representation $\mathrm{Sym}_{j}$, we can take

$$
(j-i) u_{1}^{j-i-1} u_{2}^{i} z_{1}+i u_{1}^{j-i} u_{2}^{i-1} z_{2}
$$

$(0 \leq i \leq j)$ as an equivalent basis. In the same way, for the basis $u_{1}^{j+2-i} u_{2}^{i}$ of the representation space of $\mathrm{Sym}_{j+2}$, we can take

$(j+2-i)(j+1-i) u_{1}^{j-i} u_{2}^{i} z_{1}^{2}+2(j+2-i) i u_{1}^{j+1-i} u_{2}^{i-1} z_{1} z_{2}+i(i-1) u_{1}^{j+2-i} u_{2}^{i-2} z_{2}^{2}$

as an equivalent basis by taking $z \cdot \operatorname{grad}_{u}$ twice. On the other hand, for $P(u, z)=$ $\left(z_{1} u_{2}-u_{1} z_{2}\right)^{l}$, we have $P(u A, z A)=\operatorname{det}(A)^{l} P(u, z)$. For any element $A(\tau, u)=$ $\sum_{i=0}^{j+2} a_{i}(\tau) u_{1}^{j+2-i} u_{2}^{i} \in A_{k, j+2}\left(\Gamma_{2}\right)$ and $B(\tau, u)=\sum_{i=0}^{j} b_{i}(\tau) u_{1}^{j-i} u_{2}^{j} \in A_{k+1, j}\left(\Gamma_{2}\right)$, we write

$$
\begin{aligned}
A(\tau, u, z)= & \sum_{i=0}^{j+2}\left[(j+2-i)(j+1-i) u_{1}^{j-i} u_{2}^{i} z_{1}^{2}\right. \\
& \left.+2 i(j+2-i) u_{1}^{j+1-i} u_{2}^{i-1} z_{1} z_{2}+i(i-1) u_{1}^{j+2-i} u_{2}^{i-2} z_{2}^{2}\right] a_{i}(\tau) \\
B(\tau, u, z)= & \sum_{i=0}^{j}\left[j u_{1}^{j-1-i} u_{2}^{i} z_{1}+i u_{1}^{j-i} u_{2}^{i-1} z_{2}\right] b_{i}(\tau) .
\end{aligned}
$$

If we define $\xi_{2}$ for any $f \in J_{(k, j), m}\left(\Gamma_{2}\right)$ as before, we have

$$
\xi_{2}(\tau, u, z)=A(\tau, u, z)+\left(u_{1} z_{2}-u_{2} z_{1}\right) B(\tau, u, z)+\left(u_{1} z_{2}-u_{2} z_{1}\right)^{2} C(\tau, u)
$$

with $A(\tau, u) \in A_{k, j+2}\left(\Gamma_{2}\right), B(\tau, u) \in A_{k+1, j}\left(\Gamma_{2}\right)$ and $C(\tau, u)=\sum_{i=0}^{j-2} c_{i}(\tau) u_{1}^{j-2-i} u_{2}^{i}$ $\in A_{k+2, j-2}\left(\Gamma_{2}\right)$. 
Theorem 3.5. There exists a linear mapping from $J_{(k, j), m}\left(\Gamma_{2}^{J}\right)$ to $A_{k, j}\left(\Gamma_{2}\right) \oplus$ $A_{k, j+2}\left(\Gamma_{2}\right) \oplus A_{k+1, j}\left(\Gamma_{2}\right) \oplus A_{k, j-2}\left(\Gamma_{2}\right)$ defined by

$$
f(\tau, u, z) \mapsto\left(f_{0}(\tau, u), A(\tau, u), B(\tau, u), C(\tau, u)\right)
$$

where $A(\tau, u) \in A_{k, j+2}\left(\Gamma_{2}\right), B(\tau, u) \in A_{k, j}\left(\Gamma_{2}\right), C(\tau, u) \in A_{k+2, j-2}\left(\Gamma_{2}\right)$ are uniquely determined by the decomposition

$$
\xi_{2}(\tau, u, z)=A(\tau, u, z)+\left(u_{1} z_{2}-u_{2} z_{1}\right) B(\tau, u, z)+\left(u_{1} z_{2}-u_{2} z_{1}\right)^{2} C(\tau, u) .
$$

If $m=1$, then this mapping is injective, and in particular, $f \in J_{(k, j), 1}\left(\Gamma_{2}^{J}\right)$ is a Jacobi cusp form if and only if its image under this mapping is in $S_{k, j}\left(\Gamma_{2}\right) \oplus$ $S_{k, j+2}\left(\Gamma_{2}\right) \oplus A_{k+1, j}\left(\Gamma_{2}\right) \oplus A_{k+2, j-2}\left(\Gamma_{2}\right)$.

Proof. Only the claim on Jacobi cusp forms has not been proved yet. For $f \in$ $J_{(k, j), 1}\left(\Gamma_{2}^{J}\right)$, we have $\Phi^{J}(f) \in J_{k+j, 1}\left(\Gamma_{1}^{J}\right) u_{1}^{j}$ and we know that $J_{k+j, 1}\left(\Gamma_{1}^{J}\right)$ is determined by the Taylor coefficients up to degree two, as is shown in [3]. So we check the coefficients of $u_{1}^{j}$ and $u_{1}^{j} z_{1}^{2}$ in $\Phi^{J}(f)$. By definition, denoting by $\Phi$ the usual Siegel $\Phi$ operator, the coefficient of $u_{1}^{j}$ in $\Phi^{J}(f)$ is $\Phi\left(f_{0}\right)$, and that of $u_{1}^{j} z_{1}^{2}$ is

$$
\begin{aligned}
2(2 \pi i)^{2}\left(\alpha_{1} \Phi\left(\frac{\partial \phi_{0}}{\partial \tau_{11}}\right)+\alpha_{2} j \Phi\left(\frac{\partial \phi_{0}}{\partial \tau_{11}}\right)+\alpha_{3} j(j-1) \Phi\left(\frac{\partial \phi_{0}}{\partial \tau_{11}}\right)\right) & \\
& +(j+2)(j+1) \Phi\left(a_{0}(\tau)\right),
\end{aligned}
$$

where we write $f_{0}=\sum_{i=0}^{j} \phi_{i}(\tau) u_{1}^{j-i} u_{2}^{i}$ and $A(\tau, u)=\sum_{i=0}^{j+2} a_{i}(\tau) u_{1}^{j+2-i} u_{2}^{i}$ as before. If $f$ is a cusp form, then $\Phi\left(f_{0}\right)=0$, hence $\Phi\left(\phi_{0}\right)=0$ and this also means that $\Phi\left(\frac{\partial \phi_{0}}{\partial \tau_{11}}\right)=0$. So we also have $\Phi\left(a_{0}(\tau)\right)=0$ and this means $\Phi(A(\tau, u))=0$ (see [1]). Conversely, if $f_{0} \in S_{k, j}\left(\Gamma_{2}\right)$ and $A(\tau, u) \in S_{k, j+2}\left(\Gamma_{2}\right)$, then $\Phi\left(f_{0}\right)=0$ (so $\left.\Phi\left(\frac{\partial \phi_{0}}{\partial \tau_{11}}\right)=0\right)$, and $\Phi\left(a_{0}(\tau)\right)=0$, so $\Phi^{J}(f)=0$. So $f$ is a cusp form.

\section{§4. Characterization of the image}

In this section, we assume that the index of the Jacobi forms we consider is always one. For any function $h(\tau)$ on $\mathfrak{H}_{2}$, we define the Witt operator $W$ by

$$
(W h)\left(\tau_{11}, \tau_{22}\right)=h\left(\begin{array}{cc}
\tau_{11} & 0 \\
0 & \tau_{22}
\end{array}\right)
$$

We first consider some necessary condition on the Taylor coefficients of $f \in$ $J_{(k, j), 1}\left(\Gamma_{2}^{J}\right)$. Notation being as before. we always have

$$
W\left(f_{11}\right)=0 \text {. }
$$


T. IBUKIYAMA

The reason is as follows. The Taylor coefficients satisfy the linear equation (2.6). We see directly from the definition that for any $\nu \in(\mathbb{Z} / 2 \mathbb{Z})^{2}, \vartheta_{\nu}(\tau)$ is an even function with respect to $\tau_{12}$. (Indeed, if we write $\nu=\left(\nu_{1}, \nu_{2}\right)$ and $p=\left(p_{1}, p_{2}\right)$ in the definition of $\vartheta_{\nu}(\tau)=\vartheta_{\nu}(\tau, 0)$, the change of $\tau_{12}$ to $-\tau_{12}$ is equivalent to changing $p_{1}+\nu_{1} / 2$ to $p_{1}^{\prime}+\nu_{1} / 2$ with $p_{1}^{\prime}=-\left(p_{1}+\nu_{1}\right)$.) This means that $W\left(\partial_{12} \vartheta_{\nu}(\tau)\right)=0$, so the third row of $\Theta(\tau)$ is all zero under $W$. On RHS, this means that $W\left(f_{11}\right)=0$. We see that this condition is also sufficient for the existence of $f \in J_{(k, j), 1}\left(\Gamma_{2}^{J}\right)$ for a given set $\left(f_{0}, f_{20}, f_{11}, f_{02}\right)$. When $j=0$, this is explained already in [10], so here we assume that $j \geq 2$. The more precise statement is as follows.

We assume $j \geq 2$. We take $f_{0}(\tau, z) \in A_{k, j}\left(\Gamma_{2}\right), A(\tau, u) \in A_{k, j+2}\left(\Gamma_{2}\right), B(\tau, u) \in$ $A_{k+1, j}\left(\Gamma_{2}\right), C(\tau, u) \in A_{k+2, j-2}\left(\Gamma_{2}\right)$. Sometimes we also write $F=f_{0}$ and for this $F$, we define $F_{1}, F_{2}$ by (3.2), (3.3) (we use $F$ to avoid the slightly confusing notation $\left.\left(f_{0}\right)_{1},\left(f_{0}\right)_{2}\right)$. We also define $A(\tau, u, z)$ and $B(\tau, u, z)$ by $(3.14),(3.15)$. We set

$$
\begin{aligned}
\xi_{2}(\tau, u, z)= & A(\tau, u, z)+\left(u_{1} z_{2}-u_{2} z_{1}\right) B(\tau, u, z)+\left(u_{1} z_{2}-u_{2} z_{1}\right)^{2} C(\tau, u), \\
f_{2}(\tau, u, z)= & \xi_{2}(\tau, u, z)+\alpha_{1}\left(\partial_{\tau}[z, z] F\right)(\tau, u, z) \\
& +\alpha_{2}\left(\partial_{\tau}[u, z] F_{1}\right)(\tau, u, z)+\alpha_{3}\left(\partial_{\tau}[u, u] F_{2}\right)(\tau, u, z) .
\end{aligned}
$$

Then $f_{2}$ is a polynomial in $z$ of degree two and we write it as

$$
f_{2}(\tau, u, z)=\frac{1}{2(2 \pi i)^{2}}\left(f_{20}(\tau, u) z_{1}^{2}+f_{11}(\tau, u) z_{1} z_{2}+f_{02}(\tau, u) z_{2}^{2}\right) .
$$

Theorem 4.1. Notation being as above, the functions $\left(f_{0}, f_{20}, f_{11}, f_{02}\right)$ are the Taylor coefficients of some $f(\tau, u, z) \in J_{(k, j), 1}\left(\Gamma_{2}^{J}\right)$ such that

$$
f(\tau, u, z)=f_{0}(\tau, u)+2(2 \pi i)^{2} f_{2}(\tau, u, z)+O\left(z^{4}\right)
$$

if and only if $W\left(f_{11}\right)=0$.

Proof. We have already seen the "only if" part, so we prove the converse. For the given functions $f_{0}, f_{20}, f_{11}, f_{02}$ in the theorem, we define $c_{i j}(\tau)=c_{i j}(\tau, u)$ $(0 \leq i, j \leq 1)$ as the unique solution of $(2.6)$ and define

$$
f(\tau, u, z)=\sum_{\nu \in(\mathbb{Z} / 2 \mathbb{Z})^{2}} c_{\nu}(\tau, u) \vartheta_{\nu}(\tau, z) .
$$

This is an even function of $z$ since $\vartheta_{\nu}(\tau, z)$ are so. We will show that $f(\tau, u, z)$ is the Jacobi form we want. By definition, the functions $c_{\nu}(\tau)$ are meromorphic, but we must show that they are holomorphic. First we show that the $c_{\nu}(\tau)$ are holomorphic on the standard fundamental domain $\mathcal{F}$ of $\Gamma_{2}$ in $\mathfrak{H}_{2}$. Let $\Theta(\tau)$ be as in (2.5) and denote by $B_{i j}$ the $(i, j)$-cofactor of $\Theta(\tau)$, i.e. $(-1)^{i+j}$ times the 
determinant of the $3 \times 3$ matrix obtained by deleting the $i$-th row and the $j$-th column of $\Theta(\tau)$. Then $W\left(B_{i j}\right)=0$ if $i \neq 3$ since the third row of $W(\Theta(\tau))$ is zero. Then by the assumption that $W\left(f_{11}\right)=0$, we have

$$
W\left(B_{1 i} f_{0}+\frac{1}{2(2 \pi i)^{2}}\left(B_{2 i} f_{20}+B_{3 i} f_{11}+B_{4 i} f_{02}\right)\right)=\frac{W\left(B_{3 i}\right) W\left(f_{11}\right)}{2(2 \pi i)^{2}}=0 .
$$

So every component of the vector obtained by the cofactor matrix of $\Theta(\tau)$ times ${ }^{t}\left(f_{0}, f_{20} / 2(2 \pi i)^{2}, f_{11} / 2(2 \pi i)^{2}, f_{02} / 2(2 \pi i)^{2}\right)$ is zero under $W$, and hence each $c_{i j}(\tau)$ is obtained by dividing the function which vanishes under $W$ by $\operatorname{det}(\Theta(\tau))=\chi_{5}(\tau)$. But it is known that on the fundamental domain $\mathcal{F}, \chi_{5}$ has a zero at $\tau_{12}=0$ of order one and no other zeros (see [5]). Hence the $c_{\nu}(\tau)$ are holomorphic on $\mathcal{F}$ and $f(\tau, u, z)$ is holomorphic on $\mathcal{F} \times \mathbb{C}^{2}$.

Now we show that $f(\tau, u, z)$ satisfies conditions (2.1) and (2.2) of automorphy. Since $\vartheta_{\nu}(\tau, z)$ satisfies $(2.2)$, and since (2.2) is a property of a function of $z$, the function $f(\tau, u, z)$ also satisfies (2.2). Next we check (2.1). For $\gamma=\left(\begin{array}{ll}a & b \\ c & d\end{array}\right) \in \Gamma_{2}$, we consider the Taylor expansion along $z=0$ of

$$
\left.f\right|_{(k, j), 1}[\gamma]=\operatorname{det}(c \tau+d)^{-k} e\left(-z(c \tau+d)^{-1} c^{t} z\right) f\left(\gamma \tau, u(c \tau+d)^{-1}, z(c \tau+d)^{-1}\right)
$$

Since $f=f_{0}+2(2 \pi i)^{2} f_{2}(\tau, u, z)+O\left(z^{4}\right)$, we have

$$
\begin{aligned}
& \left.f\right|_{(k, j), 1}[\gamma]=e\left(-z(c \tau+d)^{-1} c^{t} z\right)\left(\left.f_{0}\right|_{(k, j)}[\gamma]\right) \\
& +2(2 \pi i)^{2} e\left(-z(c \tau+d)^{-1} c^{t} z\right) \operatorname{det}(c \tau+d)^{-k} f_{2}\left(\gamma \tau, u(c \tau+d)^{-1}, z(c \tau+d)^{-1}\right)+O\left(z^{4}\right) .
\end{aligned}
$$

The constant term of this expansion is

$$
\left.f_{0}\right|_{(k, j)}[\gamma]=f_{0}
$$

The order two part of $\left.f\right|_{(k, j), 1}[\gamma]$ is given by

$$
\begin{aligned}
-(2 \pi i)\left(z(c \tau+d)^{-1} c^{t} z\right) f_{0}(\tau, u) & \\
& +2(2 \pi i)^{2} \operatorname{det}(c \tau+d)^{-k} f_{2}\left(\gamma \tau, u(c \tau+d)^{-1}, z(c \tau+d)^{-1}\right) .
\end{aligned}
$$

For any polynomial $h(\tau, u, z)$ in $u$ and $z$ with coefficients holomorphic in $\tau$, we write

$$
\left(\left.h\right|_{k}[\gamma]\right)(\tau, u, z)=\operatorname{det}(c \tau+d)^{-k} h\left(\gamma \tau, u(c \tau+d)^{-1}, z(c \tau+d)^{-1}\right)
$$

Then, writing $F=f_{0}$, we have

$$
\left.f_{2}\right|_{k}[\gamma]=\left.\xi_{2}\right|_{k}[\gamma]+\left.\alpha_{1}\left(\partial_{\tau}[z, z] F\right)\right|_{k}[\gamma]+\left.\alpha_{2}\left(\partial_{\tau}[u, z] F_{1}\right)\right|_{k}[\gamma]+\left.\alpha_{3}\left(\partial_{\tau}[u, u] F_{2}\right)\right|_{k}[\gamma] .
$$


By (3.13) and the definition of $\xi_{2}$, this is equal to

$$
\begin{aligned}
\xi_{2}+\alpha_{1}\left(\partial_{\tau}[z, z] F_{1}\right)+\alpha_{2}\left(\partial[u, z] F_{2}\right) & +\alpha_{3}\left(\partial_{\tau}[u, u] F_{2}\right)+\frac{1}{2(2 \pi i)}\left(z(c \tau+d)^{-1} c^{t} z\right) F \\
& =f_{2}(\tau, u, z)+\frac{1}{2(2 \pi i)}\left(z(c \tau+d)^{-1} c^{t} z\right) f_{0}(\tau, u)
\end{aligned}
$$

and hence

$$
\left.2(2 \pi i)^{2} f_{2}\right|_{k}[\gamma]-(2 \pi i)\left(z(c \tau+d)^{-1} c^{t} z\right) f_{0}(\tau, u)=2(2 \pi i)^{2} f_{2}(\tau, u, z) .
$$

This means that the Taylor expansion of $\left.f\right|_{(k, j), 1}[\gamma]$ up to order two is

$$
f_{0}(\tau, u)+2(2 \pi i)^{2} f_{2}(\tau, u, z)
$$

That is, up to order two, the Taylor expansion of $f(\tau, u, z)$ is the same as that of $\left(\left.f\right|_{(k, j), 1}\right)[\gamma]$. Now, we fix a branch of $\operatorname{det}(c \tau+d)^{1 / 2}$ and for any function $h(\tau, z)$ and any odd $l \in \mathbb{Z}$, we define

$$
\left.h\right|_{l / 2}[\gamma]=e\left(-z(c \tau+d)^{-1} c^{t} z\right)\left(\operatorname{det}(c \tau+d)^{1 / 2}\right)^{-l} h\left(\gamma \tau, z(c \tau+d)^{-1}\right) .
$$

It is well known that for any $\gamma \in \Gamma_{2}$ there exists a matrix $R(\gamma) \in \operatorname{GL}(4,(\mathbb{C})$ depending on $\gamma$ and the choice of the branch such that

$$
\left(\begin{array}{c}
\left(\left.\vartheta_{00}\right|_{1 / 2}[\gamma]\right)(\tau, z) \\
\left(\left.\vartheta_{01}\right|_{1 / 2}[\gamma]\right)(\tau, z) \\
\left(\left.\vartheta_{10}\right|_{1 / 2}[\gamma]\right)(\tau, z) \\
\left(\left.\vartheta_{11}\right|_{1 / 2}[\gamma]\right)(\tau, z)
\end{array}\right)=R(\gamma)\left(\begin{array}{l}
\vartheta_{00}(\tau, z) \\
\vartheta_{01}(\tau, z) \\
\vartheta_{10}(\tau, z) \\
\vartheta_{11}(\tau, z)
\end{array}\right)
$$

(for example, see [10, pp. 588-589]). So we have

$$
\left.f\right|_{(k, j), 1}[\gamma]=\sum_{\nu \in(\mathbb{Z} / 2 \mathbb{Z})^{2}} c_{\nu}^{\gamma}(\tau, u) \vartheta_{\nu}(\tau, z)
$$

for some meromorphic function $c_{\nu}^{\gamma}(\tau, u)$. Since the $c_{\nu}^{\gamma}(\tau, u)$ are determined only by the Taylor coefficients of $\left.f\right|_{(k, j), 1}[\gamma]$ up to degree two and since these coefficients are equal to those of $f, c_{\nu}^{\gamma}(\tau, u)=c_{\nu}(\tau, u)$ and hence $\left.f\right|_{(k, j), 1}[\gamma]=f$. For any $\tau \in \mathfrak{H}_{2}$, take $\gamma \in \Gamma_{2}$ such that $\gamma \tau \in \mathcal{F}$. Then since

$$
f(\tau, u, z)=e\left(-z(c \tau+d)^{-1} c^{t} z\right) \operatorname{det}(c \tau+d)^{-k} f\left(\gamma \tau, u(c \tau+d)^{-1}, z(c \tau+d)^{-1}\right),
$$

$f$ is holomorphic on the whole space of $(\tau, z) \in \mathfrak{H}_{2} \times \mathbb{C}^{2}$. So $f \in J_{(k, j), 1}\left(\Gamma_{2}^{J}\right)$. 


\section{$\S 5$. Dimension formulas}

\section{$\S 5.1$. The image of the mapping and dimensions}

Now, we consider the general case. We must see what the condition $W\left(f_{11}\right)=0$ exactly means in terms of Siegel modular forms. For given forms

$$
\begin{aligned}
f_{0}(\tau, u) & =F(\tau, u)=\sum_{i=0}^{j} \phi_{i}(\tau) u_{1}^{j-i} u_{2}^{i} \in A_{k, j}\left(\Gamma_{2}\right), \\
A(\tau, u) & =\sum_{i=0}^{j+2} a_{i}(\tau) u_{1}^{j+2-i} u_{1}^{i} \in A_{k, j+2}\left(\Gamma_{2}\right), \\
B(\tau, u) & =\sum_{i=0}^{j} b_{i}(\tau) u_{1}^{j-i} u_{2}^{i} \in A_{k+1, j}\left(\Gamma_{2}\right), \\
C(\tau, u) & =\sum_{i=0}^{j-2} c_{i}(\tau) u_{1}^{j-2-i} u_{2}^{i} \in A_{k+2, j-2}\left(\Gamma_{2}\right),
\end{aligned}
$$

we define $\xi_{2}$ by (4.1), $f_{2}$ by (4.2) and $f_{11}$ by (4.3) as before. Then the coefficient in $z_{1} z_{2}$ of $\xi_{2}$ is given by

$$
\sum_{i=0}^{j}\left[2(i+1)(j+1-i) a_{i+1}(\tau)+(j-2 i) b_{i}(\tau)-2 c_{i-1}(\tau)\right] u_{1}^{j-i} u_{2},
$$

where we set $c_{-1}(\tau)=c_{j-1}(\tau)=0$. The coefficient of $z_{1} z_{2}$ in $\partial_{\tau}[z, z] F$ is

$$
\sum_{i=0}^{j} \frac{\partial \phi_{i}}{\partial \tau_{12}} u_{1}^{j-i} u_{2}^{i}
$$

that in $\partial_{\tau}[u, z] F_{1}$ is

$$
\sum_{i=0}^{j} u_{1}^{j-i} u_{2}^{i}\left[\frac{j}{2} \frac{\partial \phi_{i}}{\partial \tau_{12}}+(i+1) \frac{\partial \phi_{i+1}}{\partial \tau_{11}}+(j+1-i) \frac{\partial \phi_{i-1}}{\partial \tau_{22}}\right],
$$

where we set $\phi_{-1}=\phi_{j+1}=0$, and that in $\partial_{\tau}[u, u] F_{2}$ is

$$
\sum_{i=0}^{j}\left[2(i+1)(j-i-1) \frac{\partial \phi_{i+1}}{\partial \tau_{11}}+2 i(j-i) \frac{\partial \phi_{i}}{\partial \tau_{12}}+2(i-1)(j-i+1) \frac{\partial \phi_{i-1}}{\partial \tau_{22}}\right] .
$$

To write down $f_{11}$ briefly, we prepare some notation. For $0 \leq i \leq j$, we define

$$
\begin{aligned}
h_{i}^{*}(\tau) & =\alpha_{1} \frac{\partial \phi_{i}}{\partial \tau_{12}}+\alpha_{2}\left(\frac{j}{2} \frac{\partial \phi_{i}}{\partial \tau_{12}}+(i+1) \frac{\partial \phi_{i+1}}{\partial \tau_{11}}+(j+1-i) \frac{\partial \phi_{i-1}}{\tau_{22}}\right) \\
& +\alpha_{3}\left(2(i+1)(j-i-1) \frac{\partial \phi_{i}}{\partial \tau_{11}}+2 i(j-i) \frac{\partial \phi_{i}}{\partial \tau_{12}}+2(j-i)(j-i+1) \frac{\partial \phi_{i-1}}{\partial \tau_{22}}\right),
\end{aligned}
$$


or more concretely

$$
\begin{aligned}
h_{i}^{*}(\tau)= & (k+i-1)(k+j-i-1) \frac{\partial \phi_{i}}{\partial \tau_{12}} \\
& -(k+i-1)(i+1) \frac{\partial \phi_{i+1}}{\partial \tau_{11}}-(j-i+1)(k+j-i-1) \frac{\partial \phi_{i-1}}{\partial \tau_{22}} .
\end{aligned}
$$

If we write the coefficient of $z_{1} z_{2}$ in $f_{2}$ as

$$
\frac{1}{2(2 \pi i)^{2}} f_{11}(\tau, u)=\sum_{i=0}^{j} h_{i}(\tau) u_{1}^{j-i} u_{2}^{i}
$$

then by definition,

$$
h_{i}(\tau)=h_{i}^{*}(\tau)+2(i+1)(j+1-i) a_{i+1}(\tau)+(j-2 i) b_{i}(\tau)-2 c_{i-1}(\tau) .
$$

In order to see the image $W\left(f_{11}\right)$ or $W\left(h_{i}\right)$ more concretely, we review the properties of the Witt operator on vector valued Siegel modular forms $A_{k, j}\left(\Gamma_{2}\right)$ and also on $f_{11}$. First we define the candidate for the image space of $W$ as in [14]. The function $h\left(\tau_{11}, \tau_{22}\right)$ on $\mathfrak{H}_{1} \times \mathfrak{H}_{1}$ which is an elliptic modular form of weight $k_{1}$ with respect to $\tau_{11}$ and of weight $k_{2}$ with respect to $\tau_{22}$ is identified with an element in $A_{k_{1}}\left(\Gamma_{1}\right) \otimes A_{k_{2}}\left(\Gamma_{1}\right)$. We will understand such tensor spaces in this sense. For even $k \geq 4$ we denote by $E_{k}$ the Eisenstein series of $\Gamma_{1}$ of weight $k$ and we set $E_{k}=0$ for any other $k$. For even $j \geq 2$, define

$$
\begin{aligned}
& V_{k, j}=\left\{\sum_{i=0}^{j} h_{i}\left(\tau_{11}, \tau_{22}\right) u_{1}^{j-i} u_{2}^{i} ; h_{i}\left(\tau_{11}, \tau_{22}\right) \in S_{k+j-i}\left(\Gamma_{1}\right) \otimes S_{k+i}\left(\Gamma_{1}\right),\right. \\
& \left.h_{i}\left(\tau_{22}, \tau_{11}\right)=(-1)^{k} h_{j-i}\left(\tau_{11}, \tau_{22}\right)(i=0, \ldots, j)\right\}, \\
& E_{k, j}=\left\{h\left(\tau_{11}\right) E_{k}\left(\tau_{22}\right) u_{1}^{j}+E_{k}\left(\tau_{11}\right) h\left(\tau_{22}\right) u_{2}^{j} ; h(\tau) \in S_{k+j}\left(\Gamma_{1}\right)\right\}, \\
& \widetilde{V}_{k, j}=V_{k, j}+E_{k, j} .
\end{aligned}
$$

When $j=0$, we define

$$
\widetilde{V}_{k, 0}=\left\{h\left(\tau_{11}, \tau_{22}\right) \in A_{k}\left(\Gamma_{1}\right) \otimes A_{k}\left(\Gamma_{1}\right) ; h\left(\tau_{11}, \tau_{22}\right)=h\left(\tau_{22}, \tau_{11}\right)\right\},
$$

that is, the space of symmetric tensors of $A_{k}\left(\Gamma_{1}\right)$, and define $V_{k, 0}$ to be the subspace consisting of the symmetric tensors of cusp forms. For $F=\sum_{i=0}^{\infty} \phi_{i}(\tau) u_{1}^{j-i} u_{2}^{i} \in$ $A_{k, j}\left(\Gamma_{2}\right)$, we have $W(F) \in \widetilde{V}_{k, j}$, and $F$ is a cusp form if and only if $W(F) \in$ $V_{k, j}$ (see [1] and [14]). More concretely, the elements $W\left(\phi_{i}\right)$ have the following properties for $j \geq 2$ :

$$
\begin{aligned}
& W\left(\phi_{0}\right)\left(\tau_{11}, \tau_{22}\right)=(-1)^{k} W\left(\phi_{j}\right)\left(\tau_{22}, \tau_{11}\right) \in S_{k+j}\left(\Gamma_{1}\right) \otimes A_{k}\left(\Gamma_{1}\right), \\
& W\left(\phi_{i}\right) \in S_{k+j-i}\left(\Gamma_{1}\right) \otimes S_{k+i}\left(\Gamma_{1}\right) \quad(1 \leq i \leq j-1), \\
& W\left(\phi_{j-i}\right)\left(\tau_{22}, \tau_{11}\right)=(-1)^{k} W\left(\phi_{i}\right)\left(\tau_{11}, \tau_{22}\right) \quad(0 \leq i \leq j) .
\end{aligned}
$$


For $F \in A_{k, j}\left(\Gamma_{2}\right)$, we have $F \in S_{k, j}\left(\Gamma_{2}\right)$ if and only if $W\left(\phi_{0}\right) \in S_{k+j}\left(\Gamma_{1}\right) \times S_{k}\left(\Gamma_{1}\right)$. Since there is no elliptic modular form of odd weight, we have $W\left(\phi_{i}\right)=0$ if $k+i$ is odd. Moreover, we can see by the action of $\left(\begin{array}{cccc}1 & 0 & 0 & 0 \\ 0 & -1 & 0 & 0 \\ 0 & 0 & 1 & 0 \\ 0 & 0 & 0 & -1\end{array}\right)$ that $\phi_{i}(\tau)$ is an even (resp. odd) function of $\tau_{12}$ if $i+k$ is even (resp. odd). So if $i \equiv k \bmod 2$, then

$$
W\left(\frac{\partial \phi_{i}}{\partial \tau_{12}}\right)=0
$$

and if $i \equiv k+1 \bmod 2$, then

$$
W\left(\frac{\partial \phi_{i}}{\partial \tau_{11}}\right)=W\left(\frac{\partial \phi_{i}}{\partial \tau_{22}}\right)=0 .
$$

We quote the following theorem which is later applied to obtain the dimension of $J_{(k, j), 1}\left(\Gamma_{2}^{J}\right)$.

Theorem 5.1 ([14]). For any integer $k \geq 10$ and any even integer $j \geq 0$, we have

$$
W\left(A_{k, j}\left(\Gamma_{2}\right)\right)=\widetilde{V}_{k, j} \quad \text { and } \quad W\left(S_{k, j}\left(\Gamma_{2}\right)\right)=V_{k, j} .
$$

We can also see that $W\left(f_{11}\right)$ or $W\left(h_{i}\right)$ has a similar property, as is shown in the following lemma, though $f_{11}$ itself is not a Siegel modular form in general.

Lemma 5.2. We have

$$
\begin{aligned}
& W\left(h_{i}\right)\left(\tau_{11}, \tau_{22}\right) \in A_{k+1+j-i}\left(\Gamma_{1}\right) \times A_{k+1+i}\left(\Gamma_{1}\right) \quad \text { for } i=0, \ldots, j, \\
& W\left(h_{i}\right)\left(\tau_{22}, \tau_{11}\right)=(-1)^{k} W\left(h_{j-i}\right)\left(\tau_{11}, \tau_{22}\right) .
\end{aligned}
$$

Proof. For $\gamma_{i}=\left(\begin{array}{cc}a_{i} & b_{i} \\ c_{i} & d_{i}\end{array}\right) \in \Gamma_{1}(i=1,2)$, the action of the element

$$
\iota\left(\gamma_{1}, \gamma_{2}\right)=\left(\begin{array}{cccc}
a_{1} & 0 & b_{1} & 0 \\
0 & a_{2} & 0 & b_{2} \\
c_{1} & 0 & d_{1} & 0 \\
0 & c_{2} & 0 & d_{2}
\end{array}\right) \in \Gamma_{2}
$$

on $\left(\left(\begin{array}{cc}\tau_{11} & 0 \\ 0 & \tau_{22}\end{array}\right), u, z\right)$ yields

$$
\left(\left(\begin{array}{cc}
\gamma_{1}\left(\tau_{11}\right) & 0 \\
0 & \gamma_{2}\left(\tau_{22}\right)
\end{array}\right),\left(\left(c_{i} \tau_{i i}+d_{i}\right)^{-1} z_{i}\right)_{i=1,2},\left(\left(c_{i} \tau_{i i}+d_{i}\right)^{-1} u_{i}\right)_{i=1,2}\right) .
$$

So $z_{1} z_{2}$ is mapped to $\left(c_{1} \tau_{11}+d_{1}\right)^{-1}\left(c_{2} \tau_{22}+d_{2}\right)^{-1} z_{1} z_{2}$. We apply (4.4) for $\gamma=\iota\left(\gamma_{1}, \gamma_{2}\right)$. Then the term $z(c \tau+d)^{-1} c^{t} z$ becomes $c_{1}\left(c_{1} \tau_{11}+d_{1}\right)^{-1} z_{1}^{2}+$ $c_{2}\left(c_{2} \tau_{22}+d_{2}\right)^{-1} z_{2}^{2}$ and does not contain the term $z_{1} z_{2}$. Comparing the coefficients of $z_{1} z_{2}$ in $\left.f_{2}\right|_{k}\left[\iota\left(\gamma_{1}, \gamma_{2}\right)\right]$ and $f_{2}$, we have

$$
\begin{aligned}
\left(W f_{11}\right)\left(\left(\gamma_{1} \tau_{11}, \gamma_{2} \tau_{22}\right)\right. & \left.,\left(\left(c_{i} \tau_{i i}+d_{i}\right)^{-1} u_{i}\right)_{i=1,2}\right) \\
= & \left(c_{1} \tau_{11}+d_{1}\right)^{k+1}\left(c_{2} \tau_{22}+d_{2}\right)^{k+1}\left(W f_{11}\right)\left(\tau_{11}, \tau_{22}, u\right) .
\end{aligned}
$$


So $W\left(h_{i}\right)$ is modular of weight $k+1+j-i$ for $\tau_{11}$ and of weight $k+1+i$ for $\tau_{22}$. In the same way, if we make the element

$$
\gamma=\left(\begin{array}{llll}
0 & 1 & 0 & 0 \\
1 & 0 & 0 & 0 \\
0 & 0 & 0 & 1 \\
0 & 0 & 1 & 0
\end{array}\right)
$$

act on $f_{2}$, then the term $z(c \tau+d)^{-1} c^{t} z$ in (4.4) is zero since $c=0$ for this $\gamma$. So we have

$$
f_{2}\left(\left(\begin{array}{cc}
\tau_{22} & \tau_{12} \\
\tau_{12} & \tau_{11}
\end{array}\right),\left(u_{2}, u_{1}\right),\left(z_{2}, z_{1}\right)\right)=(-1)^{k} f_{2}\left(\tau,\left(u_{1}, u_{2}\right),\left(z_{1}, z_{2}\right)\right) .
$$

Comparing the coefficients of $z_{1} z_{2}$, we obtain the assertion for $\left(W h_{i}\right)\left(\tau_{22}, \tau_{11}\right)$.

Now, we can also show that $W\left(h_{i}^{*}\right) \in A_{k+1+j-i}\left(\Gamma_{1}\right) \otimes A_{k+1+i}\left(\Gamma_{1}\right)$. It is not difficult to show this directly from the definition using the automorphy of $f_{0}(\tau, u)$. But here we have an alternative indirect proof since we have already shown that $W\left(a_{i+1}\right), W\left(b_{i}\right), W\left(c_{i-1}\right), W\left(h_{i}\right)$ belong to $A_{k+1+j-i}\left(\Gamma_{1}\right) \otimes A_{k+1+i}\left(\Gamma_{1}\right)$ and by definition $h_{i}^{*}$ is a linear combination of these forms. We note that the Fourier expansion of $W\left(\frac{\partial \phi_{i}}{\partial \tau_{12}}\right)$ is divisible by $e\left(\tau_{11}\right) e\left(\tau_{22}\right)$. Indeed, if we write the Fourier expansion of $\phi_{i}$ as $\phi_{i}(\tau)=\sum_{N \in L_{2}^{*}} c(N) e(\operatorname{tr}(N \tau))$, then

$$
\frac{\partial \phi_{i}}{\partial \tau_{12}}=2 \pi i \sum_{N \in L_{2}^{*}} n_{12} c(N) e(\operatorname{tr}(N \tau))
$$

where $n_{12}$ is the $(1,2)$ component of each $N$. The terms with $n_{12}=0$ vanish, and if $n_{12} \neq 0$, then since $N$ is positive semidefinite, both diagonal components of $N$ must be positive. So $W\left(\frac{\partial \phi_{i}}{\partial \tau_{12}}\right)$ is divisible by $e\left(\tau_{11}\right) e\left(\tau_{22}\right)$. It is obvious that $W\left(\frac{\partial \phi_{i}}{\partial \tau_{11}}\right)$ and $W\left(\frac{\partial \phi_{i}}{\partial \tau_{22}}\right)$ are divisible by $e\left(\tau_{11}\right) e\left(\tau_{22}\right)$ for $1 \leq i \leq j-1$ since $W\left(\phi_{i}\right)$ are in the tensor product of cusp form spaces for these $i$. The same is true for $W\left(\frac{\partial \phi_{0}}{\partial \tau_{22}}\right)$ and $W\left(\frac{\partial \phi_{j}}{\partial \tau_{11}}\right)$ if $j \geq 2$, since for example, we have $\phi_{0} \in S_{k+1+j}\left(\Gamma_{1}\right) \otimes A_{k+1}\left(\Gamma_{1}\right)$, and $\frac{\partial \phi_{0}}{\partial \tau_{22}}$ means that we are taking derivatives of its component in $A_{k+1}\left(\Gamma_{1}\right)$. These arguments do not work for $W\left(\frac{\partial \phi_{0}}{\partial \tau_{11}}\right)$ and $W\left(\frac{\partial \phi_{j}}{\partial \tau_{22}}\right)$, but in $h_{i}^{*}(\tau)$, no such term appears. Hence, as a whole, $W\left(h_{i}^{*}(\tau)\right)$ belongs to tensor products of cusp form spaces if $j \geq 2$.

Theorem 5.3. Assume that $j$ is even and $j \geq 2$. When $k$ is even with $k \geq 8$, we have

$$
\begin{aligned}
\operatorname{dim} J_{(k, j), 1}\left(\Gamma_{2}^{J}\right)= & \operatorname{dim} A_{k, j}\left(\Gamma_{2}\right)+\operatorname{dim} A_{k, j+2}\left(\Gamma_{2}\right)+\operatorname{dim} A_{k+1, j}\left(\Gamma_{2}\right) \\
& +\operatorname{dim} A_{k+2, j-2}\left(\Gamma_{2}\right)-\operatorname{dim} \widetilde{V}_{k+2, j-2} .
\end{aligned}
$$

When $k$ is odd with $k \geq 9$, we have 


$$
\begin{aligned}
\operatorname{dim} J_{(k, j), 1}\left(\Gamma_{2}^{J}\right) & \\
= & \operatorname{dim} A_{k, j}\left(\Gamma_{2}\right)+\operatorname{dim} A_{k, j+2}\left(\Gamma_{2}\right)+\operatorname{dim} A_{k+1, j}\left(\Gamma_{2}\right)+\operatorname{dim} A_{k+2, j-2}\left(\Gamma_{2}\right) \\
& -\operatorname{dim} V_{k+2, j-2}-\operatorname{dim} S_{k+1+j}\left(\Gamma_{1}\right) \times \operatorname{dim} A_{k+1}\left(\Gamma_{1}\right) .
\end{aligned}
$$

Proof. For $f_{0}(\tau, u)=\sum_{i=0}^{j} \phi_{i}(\tau) u_{1}^{j-i} u_{2}^{i} \in A_{k, j}\left(\Gamma_{2}\right)$, we define $h_{i}^{*}$ by (5.5). Since $W\left(\frac{\partial \phi_{i}}{\partial \tau_{11}}\right)\left(\tau_{11}, \tau_{22}\right)=(-1)^{k} W\left(\frac{\partial \phi_{j-i}}{\partial \tau_{22}}\right)\left(\tau_{22}, \tau_{11}\right)$, we see directly from the definition that $\left(W h_{i}^{*}\right)\left(\tau_{22}, \tau_{11}\right)=(-1)^{k}\left(W h_{j-i}^{*}\right)\left(\tau_{11}, \tau_{22}\right)$. First we assume that $k$ is even. Notation being as before, for $0 \leq i \leq j$ and for fixed $f_{0}$ and

$$
\begin{aligned}
& A(\tau, u)=\sum_{i=0}^{j+2} a_{i}(\tau) u_{1}^{j+2-i} u_{2}^{i} \in A_{k, j+2}\left(\Gamma_{2}\right), \\
& B(\tau, u)=\sum_{i=0}^{j} b_{i}(\tau) u_{1}^{j+1-i} u_{2}^{i} \in A_{k+1, j}\left(\Gamma_{2}\right),
\end{aligned}
$$

we set

$$
h_{i}^{\prime}(\tau)=h_{i}^{*}(\tau)+2(i+1)(j+1-i) a_{i+1}(\tau)+(j-2 i) b_{i}(\tau) .
$$

For a choice of $C(\tau, u)=\sum_{i=0}^{j-2} c_{i}(\tau) u_{1}^{j-2-i} u_{2}^{i} \in A_{k+2, j-2}\left(\Gamma_{2}\right)$, we have $h_{i}(\tau)=$ $h_{i}^{\prime}(\tau)-2 c_{i-1}(\tau)$ by definition with $c_{-1}=c_{j-1}=0$. We will show that there exists $C(\tau, u) \in A_{k+2, j-2}\left(\Gamma_{2}\right)$ such that $W\left(h_{i}\right)=0$ for all $i$ with $0 \leq i \leq j$. We show that $W\left(h_{0}^{\prime}\right)=W\left(h_{j}^{\prime}\right)=0$ for any choice of $f_{0}, A$ and $B$. Since $k$ is even, $W\left(a_{1}\right)$ and $W\left(b_{0}\right)$ are in tensor products of modular form spaces of odd weights so we have $W\left(a_{1}\right)=W\left(b_{0}\right)=0$. We also have $W\left(\frac{\partial \phi_{0}}{\partial \tau_{12}}\right)=W\left(\frac{\partial \phi_{1}}{\partial \tau_{11}}\right)=0$, so $W\left(h_{0}^{\prime}\right)=0$. Hence also $W\left(h_{j}^{\prime}\right)=0$. Thus $W\left(h_{i}^{\prime}\right) \in S_{k+1+j-i}\left(\Gamma_{1}\right) \otimes S_{k+1+i}\left(\Gamma_{1}\right)$ for any $i$ with $0 \leq i \leq j$. In the definition of $h_{i}^{\prime}$, the coefficient $(i+1)(j+1-i)$ is unchanged and $(j-2 i)$ becomes $-(j-2 i)$ if we replace $i$ by $j-i$. Since $W\left(a_{j+2-(i+1)}\right)\left(\tau_{22}, \tau_{11}\right)=$ $(-1)^{k} W\left(a_{i+1}\right)\left(\tau_{11}, \tau_{22}\right)$ and $W\left(b_{j-i}\right)\left(\tau_{22}, \tau_{11}\right)=(-1)^{k+1} W\left(b_{i}\right)\left(\tau_{11}, \tau_{22}\right)$, we have $W\left(h_{i}^{\prime}\right)\left(\tau_{22}, \tau_{11}\right)=(-1)^{k} W\left(h_{j-i}^{\prime}\right)\left(\tau_{11}, \tau_{22}\right)$. Since $(-1)^{k}=(-1)^{k+2}, W\left(h_{0}^{\prime}\right)=$ $W\left(h_{j}^{\prime}\right)=0$, and $W\left(h_{i+1}^{\prime}\right)\left(\tau_{22}, \tau_{11}\right)=(-1)^{k} W\left(h_{j-1-i}^{\prime}\right)\left(\tau_{11}, \tau_{22}\right)$, we have

$$
\sum_{i=0}^{j} W\left(h_{i}^{\prime}\right) u_{1}^{j-i} u_{2}^{i}=\sum_{i=0}^{j-2} W\left(h_{i+1}^{\prime}\right) u_{1}^{j-1-i} u_{2}^{i+1} \in u_{1} u_{2} V_{k+2, j-2} .
$$

By Theorem 5.1, we have $W\left(A_{k+2, j-2}\left(\Gamma_{2}\right)\right) u_{1} u_{2}=u_{1} u_{2} V_{k+2, j-2}$ if $k \geq 8$, and in this case there exists an element $C(\tau, u) \in A_{k+2, j-2}\left(\Gamma_{2}\right)$ such that $W\left(h_{i}^{\prime}\right)=$ $2 W\left(c_{i-1}\right)$ for all $i$ with $1 \leq i \leq j-1$. If we define $h_{i}$ and $f_{11}$ for these choices, then we have $W\left(f_{11}\right)=0$. The choice of such $C$ is up to the kernel of $W$. But for $k \geq 8$, by Theorem 5.1 we have

$$
\begin{aligned}
\operatorname{dim} \operatorname{Ker}\left(W \mid A_{k+2, j-2}\left(\Gamma_{2}\right)\right) & =\operatorname{dim} A_{k+2, j-2}\left(\Gamma_{2}\right)-\operatorname{dim} \widetilde{V}_{k+2, j-2} \\
& =\operatorname{dim} S_{k+2, j-2}\left(\Gamma_{2}\right)-\operatorname{dim} V_{k+2, j-2} .
\end{aligned}
$$


So we have the assertion of the theorem. (Actually the form $C$ chosen above should be a cusp form, but this does not matter because of the last equality above.)

Next we assume that $k$ is odd. By our assumption $k \geq 9$, we note that $W\left(A_{k+1, j}\left(\Gamma_{2}\right)\right)=\widetilde{V}_{k+1, j}$ and $W\left(A_{k+2, j-2}\left(\Gamma_{2}\right)\right)=\widetilde{V}_{k+2, j-2}$. Now fix arbitrary $f_{0}(\tau, u) \in A_{k, j}\left(\Gamma_{2}\right)$ and $A(\tau, u) \in A_{k, j+2}\left(\Gamma_{2}\right)$. We define $h_{i}^{*}$ for $f_{0}$ by $(5.5)$. In this case, $W\left(h_{0}^{*}\right)$ and $W\left(a_{1}\right)$ are not zero in general. But there exists $B(\tau, u) \in$ $A_{k+1, j}\left(\Gamma_{2}\right)$ such that $W\left(h_{0}^{*}+2(j+1) a_{1}(\tau)+j b_{0}(\tau)\right)=0$. Such a $B$ is given up to the subspace of $A_{k+1, j}\left(\Gamma_{2}\right)$ of dimension

$$
\operatorname{dim} A_{k+1, j}\left(\Gamma_{2}\right)-\operatorname{dim} S_{k+1+j}\left(\Gamma_{1}\right) \times \operatorname{dim} A_{k+1}\left(\Gamma_{1}\right) .
$$

(Note that actually, $b_{0}$ so chosen satisfies $W\left(b_{0}\right) \in S_{k+1+j}\left(\Gamma_{1}\right) \times S_{k+1}\left(\Gamma_{1}\right)$, but this does not matter as before since the image of $W\left(A_{k+1, j}\left(\Gamma_{2}\right)\right)$ by $W$ itself contains $S_{k+1+j}\left(\Gamma_{1}\right) \times A_{k+1}\left(\Gamma_{1}\right)$ and we are subtracting this.) Now, after fixing $f_{0}, A$ and $B$ as above, we can choose $C(\tau, u) \in A_{k+2, j-2}\left(\Gamma_{2}\right)$ such that $W\left(h_{i}\right)=0$ for $1 \leq i \leq j-1$. Such a $C$ is determined up to a subspace of dimension

$$
\operatorname{dim} A_{k+2, j-2}\left(\Gamma_{2}\right)-\operatorname{dim} \widetilde{V}_{k+2, j-2}
$$

Here since $k$ is odd, we have $\widetilde{V}_{k+2, j-2}=V_{k+2, j-2}$. So the whole dimension is

$$
\begin{aligned}
& \operatorname{dim} J_{(k, j), 1}\left(\Gamma_{2}^{J}\right)=\operatorname{dim} A_{k, j}\left(\Gamma_{2}\right)+\operatorname{dim} A_{k, j+2}\left(\Gamma_{2}\right)+\operatorname{dim} A_{k+1, j}\left(\Gamma_{2}\right) \\
& \quad-\operatorname{dim} S_{k+1+j}\left(\Gamma_{1}\right) \times \operatorname{dim} A_{k+1}\left(\Gamma_{2}\right)+\operatorname{dim} A_{k+2, j-2}\left(\Gamma_{2}\right)-\operatorname{dim} V_{k+2, j-2} .
\end{aligned}
$$

This proves the theorem.

Remark 5.4. Although the coefficient of $u_{1}^{j}$ in $\widetilde{V}_{k+1, j}$ is in $S_{k+1+j}\left(\Gamma_{1}\right) \times A_{k+1}\left(\Gamma_{2}\right)$, $\operatorname{dim} \widetilde{V}_{k+1, j}$ and $\operatorname{dim} V_{k+2, j-2}+\operatorname{dim} S_{k+1+j}\left(\Gamma_{1}\right) \times \operatorname{dim} A_{k+1}\left(\Gamma_{2}\right)$ are different. The reason is that the parity of $k$ is different, so the conditions determining $W\left(b_{j / 2}\right)$ and $W\left(c_{j / 2-1}\right)$ in $S_{k+1+j / 2}\left(\Gamma_{1}\right) \times S_{k+1+j / 2}\left(\Gamma_{1}\right)$ are different. Since $(-1)^{k+1}=1$ and $(-1)^{k+2}=-1$ if $k$ is odd, the former is symmetric and the latter is alternating. So we have

$$
\begin{aligned}
\operatorname{dim} V_{k+2, j-2}+\operatorname{dim} S_{k+1+j}\left(\Gamma_{1}\right) \times & \operatorname{dim} A_{k+1}\left(\Gamma_{1}\right) \\
& =\operatorname{dim} \widetilde{V}_{k+1, j}-\operatorname{dim} S_{k+1+j / 2}\left(\Gamma_{1}\right) .
\end{aligned}
$$

Remark 5.5. When $j=0$, the dimension formula has already been known for any $k$ (see [19], [10]). We have

$$
\operatorname{dim} J_{(k, 0), 1}\left(\Gamma_{2}^{J}\right)=\operatorname{dim} A_{k}\left(\Gamma_{2}\right)+\operatorname{dim} A_{k, 2}\left(\Gamma_{2}\right)- \begin{cases}0 & \text { if } k \text { is even } \\ \operatorname{dim} V_{k, 2} & \text { if } k \text { is odd }\end{cases}
$$


We note that if $k$ is odd, then $A_{k}\left(\Gamma_{2}\right)=S_{k}\left(\Gamma_{2}\right), A_{k, 2}\left(\Gamma_{2}\right)=S_{k, 2}\left(\Gamma_{2}\right)$ and $\operatorname{dim} V_{k, 2}$ $=\operatorname{dim} S_{k+1}\left(\Gamma_{1}\right)\left(\operatorname{dim} S_{k+1}\left(\Gamma_{1}\right)-1\right) / 2$. Now apparently the formula for the scalar valued case $j=0$ is very different from the formula for $j>0$. But the formula for $\operatorname{dim} A_{k, j}\left(\Gamma_{2}\right)$ is given by a sum of contributions of various conjugacy classes of $\Gamma_{2}$ in the Selberg trace formula, each of which is a polynomial function of $k$ and $j$ on the set $\left\{(k, j) ;(k, j) \equiv\left(a_{1}, a_{2}\right) \bmod 12\right\}$ for any fixed $\left(a_{1}, a_{2}\right)$. So we can define $\operatorname{dim} A_{k+2,-2}\left(\Gamma_{2}\right)$ by replacing $k$ by $k+2$ and $j$ by -2 formally in this formula. If we do so, then in this sense we can show that

$$
\operatorname{dim} A_{k+1,0}\left(\Gamma_{2}\right)+\operatorname{dim} A_{k+2,-2}\left(\Gamma_{2}\right)=0 .
$$

More precisely, we have a formula $\operatorname{dim} A_{k, j}\left(\Gamma_{2}\right)=\sum_{i=1}^{12} H_{i}(k, j)+\sum_{l=1}^{10} I_{l}(k, j)$, where $H_{i}(k, j)$ is the contribution of semisimple conjugacy classes and $I_{l}(k, j)$ is that of non-semisimple conjugacy classes for a fixed principal polynomial for each $i$ and $l$ (see [18], [21], or [13]). Then by direct calculation, we see $H_{i}(k+1,0)+$ $H_{i}(k+2,-2)=I_{l}(k+1,0)+I_{l}(k+2,-2)=0$ for all $i, l$ with $1 \leq i \leq 12$, $1 \leq l \leq 10$.

\section{$\S 5.2$. Generating functions of the dimensions}

Now we will calculate the dimensions more explicitly. Since it seems that the dimension formula for $k<3$ might be very different in nature, we only consider the case $k \geq 3$. The case $j=0$ is known. Writing $J_{(k, 0), 1}\left(\Gamma_{2}^{J}\right)=J_{k, 1}\left(\Gamma_{2}^{J}\right)$, the generating function of the dimensions is given by

$$
\sum_{k=1}^{\infty} J_{k, 1}\left(\Gamma_{2}^{J}\right) t^{k}=\frac{t^{4}+t^{6}+t^{10}+t^{12}+t^{21}+t^{27}+t^{29}+t^{35}}{\left(1-t^{4}\right)\left(1-t^{6}\right)\left(1-t^{10}\right)\left(1-t^{12}\right)}
$$

(see [19] and [10, p. 596]). For $j \geq 2$, we have the following theorem. We denote by $d_{k, j}$ the coefficient of $t^{k} s^{j}$ in the infinite series expansion of the following rational function $f_{\text {jacobi }}(t, s)$ of $t$ and $s$ along $t=s=0$ :

$$
f_{\text {jacobi }}(t, s)=\frac{f_{\text {even }}(t, s)+f_{\text {odd }}(t, s)}{\left(1-t^{4}\right)\left(1-t^{6}\right)^{2}\left(1-t^{10}\right)\left(1-s^{4}\right)\left(1-s^{6}\right)\left(1-s^{10}\right)\left(1-s^{12}\right)},
$$

where $f_{\text {even }}(t, s)$ and $f_{\text {odd }}(t, s)$ will be given below.

Then we have

Theorem 5.6. (1) Assume that $k \geq 8$ and $j \geq 2$. Then

$$
\operatorname{dim} J_{(k, j), 1}^{\text {cusp }}\left(\Gamma_{2}^{J}\right)=d_{k, j}
$$

(2) For $j=2,4$ and 6 , this is true for all $k>0$. 
Here we set

$$
\begin{aligned}
& f_{\text {even }}(t, s)=t^{4}\left(t^{4}+t^{6}+t^{8}+t^{10}+2 t^{12}+t^{14}+t^{16}-t^{20}-t^{22}\right) s^{2} \\
& +t^{4}\left(t^{4}+2 t^{6}+3 t^{8}+3 t^{10}+2 t^{12}+t^{14}-t^{18}-t^{20}\right) s^{4} \\
& +t^{4}\left(t^{4}+3 t^{6}+4 t^{8}+2 t^{10}-t^{14}-2 t^{16}-t^{18}+t^{20}+t^{22}\right) s^{6} \\
& +t^{4}\left(t^{2}+t^{4}+2 t^{6}+t^{8}+t^{10}-t^{12}-2 t^{14}-3 t^{16}-t^{18}+t^{20}+t^{22}+t^{24}\right) s^{8} \\
& +t^{4}\left(t^{2}+t^{4}+t^{6}-t^{12}-2 t^{14}-2 t^{16}-2 t^{18}+t^{22}+t^{24}\right) s^{10} \\
& +t^{4}\left(t^{2}+2 t^{4}+2 t^{6}-t^{8}-4 t^{10}-4 t^{12}-2 t^{14}-2 t^{16}-t^{18}+t^{20}+2 t^{22}\right) s^{12} \\
& +t^{4}\left(t^{2}+t^{4}-3 t^{8}-6 t^{10}-7 t^{12}-5 t^{14}-3 t^{16}+t^{18}+3 t^{20}+2 t^{22}\right) s^{14} \\
& +t^{4}\left(t^{2}+t^{4}-2 t^{6}-5 t^{8}-6 t^{10}-6 t^{12}-5 t^{14}-t^{16}+3 t^{18}+2 t^{20}\right) s^{16} \\
& +t^{4}\left(1+t^{2}-4 t^{6}-5 t^{8}-3 t^{10}-t^{12}+2 t^{16}+t^{18}-t^{20}-t^{22}\right) s^{18} \\
& +t^{4}\left(1+t^{2}-2 t^{6}-2 t^{8}-2 t^{10}+t^{14}+3 t^{16}+2 t^{18}-t^{22}-t^{24}\right) s^{20} \\
& +t^{4}\left(-t^{10}-t^{12}+t^{16}+2 t^{18}+t^{20}\right) s^{22} \\
& +t^{4}\left(1-t^{4}-2 t^{6}+2 t^{10}+2 t^{12}+t^{14}+2 t^{16}+2 t^{18}-t^{22}\right) s^{24} \\
& +t^{4}\left(1-t^{4}-t^{6}+t^{8}+3 t^{10}+3 t^{12}+3 t^{14}+2 t^{16}-t^{20}\right) s^{26} \\
& +t^{4}\left(t^{6}+t^{8}+t^{10}+2 t^{12}+2 t^{14}-t^{18}+t^{20}+t^{22}\right) s^{28} \\
& +t^{4}\left(-1+t^{4}+2 t^{6}-t^{10}-t^{12}-t^{14}-t^{16}+t^{18}+2 t^{20}+t^{22}\right) s^{30} \\
& +t^{4}\left(-t^{6}-t^{8}-t^{10}-t^{12}+t^{16}+t^{18}\right) s^{32}, \\
& f_{\text {odd }}(t, s)=t^{3}\left(t^{10}+2 t^{12}+t^{14}+t^{18}+t^{20}\right) s^{2} \\
& +t^{3}\left(t^{6}+t^{8}+2 t^{10}+2 t^{12}+2 t^{14}+t^{16}+t^{18}\right) s^{4} \\
& +t^{3}\left(t^{4}+2 t^{6}+2 t^{8}+t^{10}+t^{12}+2 t^{14}+t^{16}-t^{18}-t^{20}\right) s^{6} \\
& +t^{3}\left(t^{4}+3 t^{6}+3 t^{8}+t^{10}-t^{12}-t^{14}-t^{16}-2 t^{18}-t^{20}\right) s^{8} \\
& +t^{3}\left(t^{4}+2 t^{6}+3 t^{8}+t^{10}-t^{12}-3 t^{14}-2 t^{16}-2 t^{18}-t^{20}\right) s^{10} \\
& +t^{3}\left(t^{2}+2 t^{4}+t^{6}+t^{8}-2 t^{12}-4 t^{14}-4 t^{16}-2 t^{18}+t^{22}\right) s^{12} \\
& +t^{3}\left(t^{2}+2 t^{4}+t^{6}-3 t^{10}-5 t^{12}-5 t^{14}-4 t^{16}-4 t^{18}-t^{20}+t^{22}+t^{24}\right) s^{14} \\
& +t^{3}\left(t^{2}+t^{4}-2 t^{8}-4 t^{10}-5 t^{12}-5 t^{14}-4 t^{16}-3 t^{18}+t^{22}+2 t^{24}\right) s^{16} \\
& +t^{3}\left(t^{2}-t^{6}-3 t^{8}-3 t^{10}-4 t^{12}-5 t^{14}-2 t^{16}+2 t^{18}+3 t^{20}+t^{22}+t^{24}\right) s^{18} \\
& +t^{3}\left(t^{2}+t^{4}-t^{6}-4 t^{8}-4 t^{10}-2 t^{12}-t^{14}+4 t^{18}+4 t^{20}+2 t^{22}\right) s^{20} \\
& +t^{3}\left(-2 t^{8}-2 t^{10}-t^{12}+t^{14}+t^{18}+2 t^{20}+2 t^{22}+t^{24}\right) s^{22} \\
& +t^{3}\left(-2 t^{8}-2 t^{10}+2 t^{14}+3 t^{16}+3 t^{18}+2 t^{20}\right) s^{24} \\
& +t^{3}\left(-t^{8}+t^{12}+2 t^{14}+3 t^{16}+4 t^{18}+2 t^{20}-t^{24}\right) s^{26} \\
& +t^{3}\left(t^{8}+t^{10}+t^{12}+t^{14}+2 t^{16}+2 t^{18}+t^{20}-t^{24}\right) s^{28} \\
& +t^{3}\left(t^{8}+t^{10}+t^{12}+t^{14}-t^{18}-t^{20}\right) s^{30} \\
& +t^{3}\left(1-t^{4}-2 t^{6}+t^{10}+t^{12}+t^{14}+t^{16}-t^{18}-2 t^{20}-t^{22}\right) s^{32} \text {. }
\end{aligned}
$$


We will prove Theorem 5.6 in the rest of this section, based on Theorem 5.3 and (5.8). We assume that $j \geq 2$ and even, and $k \geq 3$. For this calculation, we use the already known formulas for $\operatorname{dim} A_{k, j}\left(\Gamma_{2}\right)$ ([18]) and formulas for $\operatorname{dim} V_{k, j}$ derived from classical formulas. We prove everything by using generating functions, since this is more appealing for future use than just comparing formulas term by term. To change the known formulas to generating functions is a routine but a tedious work. We explain some part of the calculations below.

First we start from generating functions related to elliptic modular forms, such as $V_{k, j}$. Note that the coefficient of $\left(u_{1} u_{2}\right)^{j / 2}$ in elements of $V_{k, j}$ is a symmetric tensor if $k$ is even and an alternating tensor if $k$ is odd. Also the coefficient of $u_{1}^{i} u_{2}^{j-i}$ is determined by the coefficient of $u_{1}^{j-i} u_{2}^{i}$. So we have the following formula for even $j \geq 2$. When $k$ is even and $j \geq 4$, we have

$$
\begin{aligned}
& \operatorname{dim} V_{k+2, j-2}=\frac{1}{2} \sum_{i=0}^{j-2} \operatorname{dim} S_{k+j-i}\left(\Gamma_{1}\right) \times \operatorname{dim} S_{k+2+i}\left(\Gamma_{1}\right)+\frac{1}{2} \operatorname{dim} S_{k+1+j / 2}\left(\Gamma_{1}\right), \\
& \operatorname{dim} \widetilde{V}_{k+2, j-2}=\operatorname{dim} V_{k+2, j-2}+\operatorname{dim} S_{k+j}\left(\Gamma_{1}\right) .
\end{aligned}
$$

When $k$ is odd, we have

$$
\begin{aligned}
\operatorname{dim} V_{k+1, j} & =\frac{1}{2} \sum_{i=0}^{j} \operatorname{dim} S_{k+1+j-i}\left(\Gamma_{1}\right) \times \operatorname{dim} S_{k+1+i}\left(\Gamma_{1}\right)+\frac{1}{2} \operatorname{dim} S_{k+1+j / 2}\left(\Gamma_{1}\right), \\
\operatorname{dim} \widetilde{V}_{k+1, j} & =\operatorname{dim} V_{k+1, j}+\operatorname{dim} S_{k+1+j}\left(\Gamma_{1}\right) .
\end{aligned}
$$

For even $k \geq 0$, we have

$$
\operatorname{dim} S_{k}\left(\Gamma_{1}\right)=\frac{k-1}{12}+\frac{1}{4}(-1)^{k / 2}+\frac{1}{3}[1,0,-1 ; 3]_{k}-\frac{1}{2}+\delta_{k 2},
$$

where $\delta_{k 2}$ is the Kronecker delta and $\left[i_{0}, i_{1}, i_{2} ; 3\right]_{k}$ means $i_{\nu}$ if $k \equiv \nu \bmod 3$. So, the generating functions of dimensions of $\widetilde{V}_{k, j}$ can be in principle calculated from this formula for $j \geq 2$. On the other hand, if $j=0$, then it is well known that

$$
\sum_{k=0}^{\infty} \widetilde{V}_{k, 0} t^{k}=\frac{1}{\left(1-t^{4}\right)\left(1-t^{6}\right)\left(1-t^{12}\right)},
$$

so for $k \geq 4$, we have

$$
\sum_{k \geq 4}^{\infty} \operatorname{dim} \widetilde{V}_{k, 0} t^{k}=\frac{t^{4}+t^{6}-t^{10}+t^{12}-t^{16}-t^{18}+t^{22}}{\left(1-t^{4}\right)\left(1-t^{6}\right)\left(1-t^{12}\right)}
$$

Then the really necessary formula for even $k \geq 4$ with $j \geq 2$ is 


$$
\begin{aligned}
& \sum_{k \geq 4, k: \text { even, } j \geq 2} \operatorname{dim}\left(\widetilde{V}_{k+2, j-2}\right) t^{k} s^{j} \\
& \quad=\frac{f_{\text {witteven }}(t, s)}{\left(1-t^{4}\right)\left(1-t^{6}\right)\left(1-t^{12}\right)\left(1-s^{2}\right)\left(1-s^{6}\right)\left(1-s^{8}\right)\left(1-s^{12}\right)} .
\end{aligned}
$$

where

$$
\begin{aligned}
& f_{\text {witteven }}=t^{4}\left(1+t^{2}+t^{6}-t^{8}-t^{12}-t^{14}+t^{20}\right) s^{2} \\
& +t^{4}\left(-1-t^{2}+t^{4}-t^{6}+t^{8}+t^{10}+2 t^{12}+t^{14}-t^{16}-t^{20}\right) s^{4} \\
& +t^{4}\left(t^{2}+t^{6}-t^{10}-t^{12}+t^{16}\right) s^{6}+t^{4}\left(-2 t^{2}-t^{4}-t^{6}+2 t^{8}+t^{10}+t^{12}+t^{14}-t^{20}\right) s^{8} \\
& +t^{4}\left(-1+2 t^{2}+t^{4}-2 t^{8}-t^{10}-t^{14}+t^{20}\right) s^{10}+t^{4}\left(2-t^{2}-2 t^{4}+t^{8}-2 t^{12}+t^{16}\right) s^{12} \\
& +t^{4}\left(-1+t^{4}-t^{6}-t^{8}+t^{12}-t^{16}\right) s^{14} \\
& +t^{4}\left(1+t^{2}+t^{6}-t^{8}-t^{10}-2 t^{12}-t^{14}+t^{20}\right) s^{16} \\
& +t^{4}\left(-t^{2}-t^{6}+t^{10}+t^{12}-t^{16}\right) s^{18}+t^{4}\left(-1+2 t^{2}+t^{4}+t^{6}-t^{8}-t^{10}-t^{14}\right) s^{20} \\
& +t^{4}\left(2-t^{2}-t^{4}-t^{6}+t^{8}+t^{10}-t^{12}+t^{18}\right) s^{22} \\
& +t^{4}\left(-2+t^{4}+t^{6}+2 t^{12}+t^{14}-t^{18}-t^{20}\right) s^{24} \\
& +t^{4}\left(1-t^{4}-t^{6}+t^{8}-t^{12}+t^{16}+t^{18}\right) s^{26}+t^{4}\left(-1-t^{2}+t^{6}+t^{12}+t^{14}-t^{18}\right) s^{28} \\
& +t^{4}\left(1+t^{2}-t^{6}-t^{8}-t^{12}-t^{14}+t^{18}+t^{20}\right) s^{30}
\end{aligned}
$$

To calculate the necessary quantities for odd $k$, we have for example

$$
\sum_{\substack{k \geq 3, k: \text { odd } \\ j \geq 2, j: \text { even }}} \operatorname{dim} S_{k+1+j / 2}\left(\Gamma_{1}\right) t^{k} s^{j}=\frac{f_{\text {mid }}(t, s)}{\left(1-t^{4}\right)\left(1-t^{6}\right)\left(1-s^{8}\right)\left(1-s^{12}\right)},
$$

where

$$
f_{\text {mid }}(t, s)=t^{9} s^{4}+t^{7} s^{8}+\left(t^{5}-t^{9}\right) s^{12}+\left(t^{3}-t^{7}-t^{9}\right) s^{16}-t^{11} s^{20} .
$$

Then we have

$$
\begin{aligned}
\sum_{\substack{k \geq 3, k: \text { odd } \\
j \geq 2, j: \text { even }}}\left(\operatorname{dim} \widetilde{V}_{k+1, j}-\operatorname{dim} S_{k+1+j / 2}\left(\Gamma_{1}\right)\right) t^{k} s^{j} \\
\quad=\frac{f_{\text {wittodd }}(t, s)}{\left(1-t^{4}\right)\left(1-t^{6}\right)\left(1-t^{12}\right)\left(1-s^{2}\right)\left(1-s^{6}\right)\left(1-s^{8}\right)\left(1-s^{12}\right)},
\end{aligned}
$$

where we set

$$
\begin{aligned}
f_{\text {wittodd }}(t, s)= & t^{3}\left(t^{6}+t^{12}+t^{14}-t^{18}\right) s^{2}+t^{3}\left(t^{4}-t^{6}+t^{8}-t^{12}-t^{14}+2 t^{18}\right) s^{4} \\
& +t^{3}\left(t^{2}-t^{4}+t^{10}+t^{12}-t^{18}\right) s^{6}+t^{3}\left(1-t^{2}-t^{10}-2 t^{12}+t^{16}+t^{18}\right) s^{8} \\
& +t^{3}\left(-1+t^{2}-t^{8}+t^{10}+t^{12}-t^{14}-t^{16}-t^{18}\right) s^{10} \\
& +t^{3}\left(1-t^{2}-t^{10}-t^{12}+t^{14}\right) s^{12}
\end{aligned}
$$




$$
\begin{aligned}
& +t^{3}\left(-1+t^{4}-t^{8}-t^{14}-t^{16}\right) s^{14}+t^{3}\left(t^{2}-t^{4}+t^{12}-t^{18}\right) s^{16} \\
& +t^{3}\left(-t^{2}+t^{4}-t^{10}-t^{12}+t^{18}\right) s^{18}+t^{3}\left(t^{2}-t^{4}+t^{10}+t^{12}-t^{18}\right) s^{20} \\
& +t^{3}\left(1-t^{2}+t^{8}-t^{10}-t^{12}+t^{14}+t^{16}+t^{18}\right) s^{22} \\
& +t^{3}\left(-1+t^{2}+t^{4}-t^{8}+t^{10}+t^{12}-t^{14}-t^{16}+t^{20}\right) s^{24} \\
& +t^{3}\left(1-t^{4}-t^{6}+t^{8}-t^{12}+t^{16}+t^{18}\right) s^{26}-t^{11} s^{28} .
\end{aligned}
$$

Finally we give the generating function of $\operatorname{dim} A_{k, j}\left(\Gamma_{2}\right)$ for $k \geq 3$. This is based on the formula for $\operatorname{dim} S_{k, j}\left(\Gamma_{2}\right)$ for $k \geq 5$ in [18] by Tsushima. I was informed that the same formula was proved for $k=4$ by C. Faber and for $k=3$ by D. Petersen. We have $A_{k, j}\left(\Gamma_{2}\right)=S_{k, j}\left(\Gamma_{2}\right)$ for odd $k$. For even $k$, by using the surjectivity of the Siegel $\Phi$ operator in [1], [16] for $k \geq 6$ and in [14] for $k=4$, we can calculate the difference from $A_{k, j}\left(\Gamma_{2}\right)$. For $j \geq 2$, it is $\operatorname{dim} S_{k+j}\left(\Gamma_{1}\right)$ and for $j=0$ it is $\operatorname{dim} A_{k}\left(\Gamma_{1}\right)$. Considering all these, we have the following formula:

$$
\begin{aligned}
& \sum_{k=3}^{\infty} \sum_{j=0}^{\infty} \operatorname{dim} A_{k, j}\left(\Gamma_{2}\right) t^{k} s^{j} \\
& \quad=\frac{f_{\text {evensiegel }}(t, s)+f_{\text {oddsiegel }}(t, s)}{\left(1-t^{4}\right)\left(1-t^{6}\right)\left(1-t^{10}\right)\left(1-t^{12}\right)\left(1-s^{6}\right)\left(1-s^{8}\right)\left(1-s^{10}\right)\left(1-s^{12}\right)},
\end{aligned}
$$

where

$$
\begin{aligned}
& f_{\text {oddsiegel }}(t, s)=t^{35}+\left(t^{21}+t^{23}+t^{27}+t^{29}-t^{33}\right) s^{2}+\left(t^{15}+t^{17}+t^{19}+t^{21}+t^{23}\right) s^{4} \\
& +\left(t^{11}+t^{13}+t^{15}+t^{17}+t^{19}+t^{21}+t^{23}-t^{35}\right) s^{6} \\
& +\left(t^{9}+t^{11}+t^{13}+2 t^{15}+2 t^{17}+t^{19}-t^{21}-t^{27}-t^{29}+t^{33}-t^{35}\right) s^{8} \\
& +\left(t^{9}+t^{11}+t^{13}+2 t^{15}+2 t^{17}+t^{19}-t^{21}-t^{23}-2 t^{27}-2 t^{29}+t^{33}-t^{35}\right) s^{10} \\
& +\left(t^{7}+2 t^{9}+2 t^{11}+t^{13}-t^{19}-3 t^{21}-3 t^{23}-t^{27}-t^{29}+t^{33}-t^{35}\right) s^{12} \\
& +\left(t^{7}+t^{9}+t^{11}+t^{13}-t^{17}-2 t^{19}-3 t^{21}-4 t^{23}-t^{25}-2 t^{27}-t^{29}+t^{33}+t^{35}\right) s^{14} \\
& +\left(t^{7}+t^{9}+t^{11}+t^{13}-2 t^{15}-3 t^{17}-4 t^{19}-3 t^{21}-4 t^{23}-2 t^{25}+t^{29}+t^{31}+t^{35}\right) s^{16} \\
& +\left(t^{5}+t^{7}-t^{11}-t^{13}-3 t^{15}-4 t^{17}-3 t^{19}-2 t^{21}-2 t^{23}-t^{25}+t^{27}+t^{29}+2 t^{35}\right) s^{18} \\
& +\left(t^{5}+t^{7}-t^{9}-t^{11}-t^{13}-3 t^{15}-5 t^{17}-4 t^{19}-t^{25}+2 t^{27}+3 t^{29}+t^{31}-t^{33}+t^{35}\right) s^{20} \\
& +\left(-t^{11}-2 t^{13}-2 t^{15}-2 t^{17}-2 t^{19}-t^{21}+t^{23}+2 t^{27}+2 t^{29}+t^{31}+t^{35}\right) s^{22} \\
& +\left(t^{5}-t^{9}-2 t^{11}-2 t^{13}-t^{15}-2 t^{17}-t^{19}+2 t^{21}+4 t^{23}\right. \\
& \left.+\left(-t^{11}-t^{13}+t^{21}+3 t^{23}+t^{25}+t^{27}+t^{29}+t^{31}-t^{35}\right) s^{26}+t^{31}-t^{33}-t^{35}\right) s^{24} \\
& +\left(-t^{11}-t^{13}+t^{15}+t^{17}+t^{19}+t^{21}+3 t^{23}+2 t^{25}-t^{35}\right) s^{28} \\
& +\left(t^{15}+t^{17}+t^{19}+t^{21}+t^{23}+t^{25}-t^{35}\right) s^{30}+\left(t^{17}+2 t^{19}+t^{21}+t^{25}-t^{29}-t^{31}\right) s^{32} \\
& +\left(t^{11}+t^{13}-t^{23}\right) s^{34}+\left(t^{3}-t^{7}-t^{9}-t^{15}+t^{17}+2 t^{19}+t^{21}-t^{23}-t^{29}-t^{31}+t^{35}\right) s^{36}
\end{aligned}
$$


and

$$
\begin{aligned}
& f_{\text {evensiegel }}(t, s) \\
& =1+\left(t^{10}+t^{14}+2 t^{16}+t^{18}-t^{20}-t^{26}-t^{28}+t^{32}\right) s^{2}+\left(t^{8}+t^{10}+t^{12}+t^{14}+t^{16}\right) s^{4} \\
& +\left(-1+t^{6}+t^{8}+t^{10}+t^{12}+t^{14}+t^{16}+t^{18}\right) s^{6} \\
& +\left(-1+t^{4}+t^{8}+t^{10}+2 t^{12}-t^{16}+t^{20}+t^{26}+t^{28}-t^{32}\right) s^{8} \\
& +\left(-1+t^{6}+t^{12}+t^{14}-t^{16}+2 t^{20}-t^{24}+t^{28}-t^{32}\right) s^{10} \\
& +\left(-1+t^{4}+t^{6}-t^{10}+t^{12}-t^{14}-3 t^{16}-2 t^{18}+t^{20}+t^{26}+t^{28}-t^{32}\right) s^{12} \\
& +\left(1-t^{8}-t^{10}-t^{12}-t^{14}-2 t^{16}-t^{18}-t^{22}-t^{24}\right) s^{14} \\
& +\left(1-t^{10}-2 t^{12}-2 t^{14}-t^{16}-2 t^{18}-3 t^{20}-t^{22}-t^{28}+t^{32}\right) s^{16} \\
& +\left(2-t^{4}-t^{6}-2 t^{12}-2 t^{18}-4 t^{20}-2 t^{22}-t^{24}-2 t^{26}-t^{28}+t^{30}+2 t^{32}\right) s^{18} \\
& +\left(1-t^{4}+t^{8}+t^{10}-2 t^{12}-t^{14}+t^{16}-2 t^{18}-5 t^{20}-2 t^{22}-t^{26}-t^{28}+t^{30}+2 t^{32}\right) s^{20} \\
& +\left(1-t^{4}-t^{6}+t^{8}+t^{10}+t^{14}+2 t^{16}-t^{18}-4 t^{20}\right. \\
& +\left(-1+t^{6}+t^{8}+t^{10}-t^{18}-t^{20}+t^{28}+t^{30}\right) s^{24} \\
& +\left(-1+t^{8}+t^{10}+2 t^{12}-t^{22}+t^{26}+t^{28}+t^{34}\right) s^{26} \\
& \left.+\left(-1+t^{4}+t^{6}-t^{8}+t^{12}-2 t^{16}+t^{18}+3 t^{20}+t^{22}+t^{26}+2 t^{28}-t^{32}\right) s^{28}+t^{30}+2 t^{32}+t^{34}\right) s^{22} \\
& +\left(-1+t^{4}+t^{12}-t^{14}-t^{16}+2 t^{20}+t^{22}+t^{24}+2 t^{26}+t^{28}-t^{32}\right) s^{30} \\
& +\left(t^{4}+t^{6}-t^{8}-2 t^{10}-t^{12}-2 t^{16}+3 t^{20}+3 t^{22}+t^{24}+t^{26}+t^{28}-t^{32}-t^{34}\right) s^{32} \\
& +\left(-t^{14}+t^{24}+t^{26}\right) s^{34}+\left(1-t^{10}-2 t^{12}+2 t^{22}+t^{24}-t^{34}\right) s^{36} \\
& +
\end{aligned}
$$

Proof of Theorem 5.6. By using the generating functions given above, the generating function for $\operatorname{dim} J_{(k, j), 1}\left(\Gamma_{2}^{J}\right)$ is obtained for $k \geq 8$ for any $j \geq 2$ by Theorem 5.3. If $k$ is odd, then $J_{(k, j), 1}^{\text {cusp }}\left(\Gamma_{2}^{J}\right)=J_{(k, j), 1}\left(\Gamma_{2}^{J}\right)$. If $k$ is even and $k \geq 6$ and $j \geq 2$, then

$$
\operatorname{dim} J_{(k, j), 1}^{\text {cusp }}\left(\Gamma_{2}^{J}\right)=\operatorname{dim} J_{(k, j), 1}\left(\Gamma_{2}\right)-\operatorname{dim} J_{k+j, 1}^{\text {cusp }}\left(\Gamma_{1}\right) .
$$

Here

$$
\sum_{\substack{k \geq 2, j \geq 2 \\ k, j: \text { even }}}^{\infty} \operatorname{dim} J_{k+j, 1}^{\text {cusp }}\left(\Gamma_{1}^{J}\right) t^{k} s^{j}=\frac{t^{8} s^{2}+\left(t^{6}-t^{8}\right) s^{4}+\left(t^{4}-t^{6}\right) s^{6}-t^{10} s^{8}}{\left(1-t^{2}\right)\left(1-t^{6}\right)\left(1-s^{2}\right)\left(1-s^{6}\right)} .
$$

By using this, we obtain the generating function of $\operatorname{dim} J_{(k, j), 1}^{\text {cusp }}\left(\Gamma_{2}^{J}\right)$. Assertion (2) for small $j$ will be proved in Proposition 5.10 in the next section.

Remark 5.7. Of course we can give the generating function of $\operatorname{dim} J_{(k, j), 1}\left(\Gamma_{2}^{J}\right)$, including non-cusp forms, as explained above. But we omit it here, since the result 
is lengthy and the only necessary calculation is to add (5.14) to the generating function for cusp forms, which everyone can do.

Remark 5.8. The above value $d_{k, j}$ coincides with Tsushima's conjecture in [20] on dimensions for all $k \geq 3$ and $j \geq 2$, so we proved his conjecture for $k \geq 8$ for index one. His conjecture includes the case of any indices and also the case of skew holomorphic Jacobi forms. We do not know the module structures in such cases in general, but see [10] for the case $m=2, j=0$.

Finally we recall the relation between Jacobi forms of index one and Siegel modular forms of half-integral weight. Let $\chi$ be the character $\Gamma_{0}^{(2)}(4)$ given by $\left(\frac{-4}{\operatorname{det}(D)}\right)$ or the trivial character. Let $A_{k-1 / 2, j}^{+}\left(\Gamma_{0}^{(2)}(4), \chi\right)$ be the plus subspace of $A_{k-1 / 2, j}\left(\Gamma_{0}^{(2)}(4), \chi\right)$ (see for example [9] or [12] for the definition). Let $S_{k-1 / 2, j}^{+}\left(\Gamma_{0}^{(2)}(4), \chi\right)$ be the subspace of cusp forms. Since we know that

$$
\begin{aligned}
& J_{(k, j), 1}\left(\Gamma_{2}^{J}\right) \cong \begin{cases}A_{k-1 / 2, j}^{+}\left(\Gamma_{0}^{(2)}(4)\right) & \text { if } k \text { is even, } \\
A_{k-1 / 2, j}^{+}\left(\Gamma_{0}^{(2)}(4), \psi\right) & \text { if } k \text { is odd },\end{cases} \\
& J_{(k, j), 1}^{\text {cusp }}\left(\Gamma_{2}^{J}\right) \cong \begin{cases}S_{k-1 / 2, j}^{+}\left(\Gamma_{0}^{(2)}(4)\right) & \text { if } k \text { is even, } \\
S_{k-1 / 2, j}^{+}\left(\Gamma_{0}^{(2)}(4), \psi\right) & \text { if } k \text { is odd },\end{cases}
\end{aligned}
$$

we have the following corollary.

Corollary 5.9. For arbitrary even $j \geq 0$, the dimensions of $A_{k-1 / 2, j}^{+}\left(\Gamma_{0}^{(2)}(4)\right)$ and $S_{k-1 / 2, j}^{+}\left(\Gamma_{0}^{(2)}(4)\right)$ for even $k$ and of $A_{k-1 / 2, j}^{+}\left(\Gamma_{0}^{(2)}(4), \psi\right)$ and $S_{k-1 / 2, j}^{+}\left(\Gamma_{0}^{(2)}(4), \psi\right)$ for odd $k$ are given explicitly by Theorem 5.6 and (5.13) for $k \geq 8$.

\section{$\S 5.3$. Remarks on small $j$}

When $j=2, j=4$ or $j=6$, we have $\operatorname{dim} J_{(k, j), 1}\left(\Gamma_{2}^{J}\right)=d_{k, j}$ also for all $k$ with $k \leq 7$.

Proposition 5.10. The dimension of $J_{k, j}\left(\Gamma_{2}^{J}\right)$ for each $k \leq 7$ and $j \leq 6$ is given in the following table:

\begin{tabular}{|c|cccccccc|}
\hline$j \backslash k$ & 0 & 1 & 2 & 3 & 4 & 5 & 6 & 7 \\
\hline 2 & 0 & 0 & 0 & 0 & 0 & 0 & 0 & 0 \\
4 & 0 & 0 & 0 & 0 & 0 & 0 & 1 & 0 \\
6 & 0 & 0 & 0 & 0 & 1 & 0 & 1 & 1 \\
\hline
\end{tabular}

and this coincides with $d_{k, j}$ defined in Theorem 5.6. 
Proof. We have the following table of dimensions:

\begin{tabular}{|c|cccccccccc|}
\hline$k$ & 0 & 1 & 2 & 3 & 4 & 5 & 6 & 7 & 8 & 9 \\
\hline $\operatorname{dim} A_{k}$ & 0 & 0 & 0 & 0 & 1 & 0 & 1 & 0 & 1 & 0 \\
$\operatorname{dim} A_{k, 2}$ & 0 & 0 & 0 & 0 & 0 & 0 & 0 & 0 & 0 & 0 \\
$\operatorname{dim} A_{k, 4}$ & 0 & 0 & 0 & 0 & 0 & 0 & 0 & 0 & 1 & 0 \\
$\operatorname{dim} A_{k, 6}$ & 0 & 0 & 0 & 0 & 0 & 0 & 1 & 0 & 1 & 0 \\
$\operatorname{dim} A_{k, 8}$ & 0 & 0 & 0 & 0 & 1 & 0 & 0 & 0 & 2 & 1 \\
\hline
\end{tabular}

(see [11] for small $k$ ). If we set

$$
a[k, j]=\operatorname{dim} A_{k, j}\left(\Gamma_{2}\right)+\operatorname{dim} A_{k, j+2}\left(\Gamma_{2}\right)+\operatorname{dim} A_{k+1, j}\left(\Gamma_{2}\right)+\operatorname{dim} A_{k+2, j-2}\left(\Gamma_{2}\right),
$$

then among $(k, j)$ with $k \leq 7$ and $j \leq 6$, only the following cases are non-zero: $a[2,2]=1, a[4,2]=1, a[6,2]=1, a[6,4]=1, a[7,4]=1, a[4,6]=1, a[5,6]=1$, $a[6,6]=2, a[7,6]=1$. For $j=2$, the dimension 1 comes from $A_{k+2,0}\left(\Gamma_{2}\right)$ in the Taylor expansion for $k=2,4,6$. This is the "C" part of $\xi_{2}$ in the Taylor expansion and we need $W(C)=0$. But no image of the Eisenstein series of weight $4,6,8$ under the Witt operator vanishes, so we have no corresponding Jacobi forms. For $(k, j)=(6,4)$, if we take $A(\tau, u)=\sum_{i=0}^{6} a_{i}(\tau) u_{1}^{6-i} u_{2}^{i} \in A_{6,6}\left(\Gamma_{2}\right)$, we have $W\left(a_{2}\right) \in S_{10}\left(\Gamma_{1}\right) \oplus S_{8}\left(\Gamma_{1}\right)=0$, so the condition is satisfied and $\operatorname{dim} J_{6,4}\left(\Gamma_{2}^{J}\right)=1$. For $(k, j)=(7,4)$, we have $A_{8,4}\left(\Gamma_{2}\right) \neq 0$. This is an Eisenstein series of Klingen type and its image under the Witt operator does not vanish, so $J_{7,4}\left(\Gamma_{2}^{J}\right)=0$. For $(k, j)=(4,6), A_{4,8}\left(\Gamma_{2}\right) \neq 0$. By checking the weight $S_{4+6-i}\left(\Gamma_{1}\right) \otimes S_{4+i}\left(\Gamma_{1}\right)$, the Witt operator vanishes for every coefficient of $u_{1}^{8-i} u_{2}^{i}$ with $i \neq 0,8$, so the vanishing condition is satisfied and we have $\operatorname{dim} J_{4,6}\left(\Gamma_{2}^{J}\right)=1$. For $(k, j)=(5,6)$, $A_{6,6}\left(\Gamma_{2}\right) \neq 0$. This is its Klingen-type Eisenstein series and its image under $W$ does not vanish, so $J_{5,6}\left(\Gamma_{2}\right)=0$. For $(k, j)=(6,6)$, we have $\operatorname{dim} A_{6,6}\left(\Gamma_{2}\right)=1$ and $\operatorname{dim} A_{8,4}\left(\Gamma_{2}\right)=1$. It is easy to see that the Witt map on $A_{8,4}\left(\Gamma_{2}\right)$ is surjective with kernel is zero. Hence, adjusting the Witt image coming from $A_{6,6}\left(\Gamma_{2}\right)$ by $A_{8,4}\left(\Gamma_{2}\right)$, we have $\operatorname{dim} J_{6,6}\left(\Gamma_{2}^{J}\right)=1$. For $(k, j)=(7,6)$, the Taylor coefficient comes from $A_{8,6}\left(\Gamma_{2}\right)$. The image of the Witt operator is zero because $V_{8,6}=0$. So there exists a Jacobi form.

For the readers' convenience, we write down the generating functions for $j=2$ explicitly below. The other cases can be also easily read off from the general generating function (5.10).

$$
\sum_{k=1}^{\infty} \operatorname{dim} J_{(k, 2), 1}\left(\Gamma_{2}^{J}\right) t^{k}=\frac{2 t^{8}+2 t^{10}+t^{12}+t^{16}}{\left(1-t^{4}\right)\left(1-t^{6}\right)^{2}\left(1-t^{10}\right)}+\frac{t^{13}+2 t^{15}+t^{17}+t^{21}+t^{23}}{\left(1-t^{4}\right)\left(1-t^{6}\right)^{2}\left(1-t^{10}\right)},
$$




$$
\sum_{k=2: \text { even }}^{\infty} \operatorname{dim} J_{(k, 2), 1}^{\text {cusp }}\left(\Gamma_{2}^{J}\right) t^{k}=\frac{t^{8}+t^{10}+t^{12}+t^{14}+2 t^{16}+t^{18}+t^{20}-t^{24}-t^{26}}{\left(1-t^{4}\right)\left(1-t^{6}\right)^{2}\left(1-t^{10}\right)} .
$$

Note that if $k$ is odd, then any Jacobi form is a cusp form, so we need not give the dimensions of cusp forms separately. Several explicit examples of Jacobi forms in $J_{(k, 2), 1}\left(\Gamma_{2}^{J}\right)$ have been given in [12].

Finally we give the numerical tables of dimensions for several small $k$ and $j$.

Table 1. $\operatorname{dim} J_{(k, j), 1}\left(\Gamma_{2}^{J}\right)$. (The values for $k \leq 7$ are conjectural for $j \geq 8$.)

\begin{tabular}{|c|ccccccccccccccccccc|}
\hline$j \backslash k$ & 3 & 4 & 5 & 6 & 7 & 8 & 9 & 10 & 11 & 12 & 13 & 14 & 15 & 16 & 17 & 18 & 19 & 20 & 21 \\
\hline 0 & 0 & 1 & 0 & 1 & 0 & 1 & 0 & 3 & 0 & 3 & 0 & 4 & 0 & 7 & 0 & 7 & 0 & 9 & 1 \\
2 & 0 & 0 & 0 & 0 & 0 & 2 & 0 & 2 & 0 & 3 & 1 & 6 & 2 & 8 & 2 & 10 & 4 & 16 & 7 \\
4 & 0 & 0 & 0 & 1 & 0 & 2 & 1 & 3 & 1 & 6 & 3 & 9 & 5 & 12 & 7 & 18 & 11 & 24 & 16 \\
6 & 0 & 1 & 0 & 1 & 1 & 3 & 2 & 6 & 3 & 9 & 6 & 13 & 10 & 20 & 14 & 26 & 20 & 35 & 28 \\
8 & 0 & 1 & 0 & 2 & 1 & 5 & 4 & 8 & 5 & 12 & 10 & 20 & 16 & 27 & 21 & 36 & 31 & 50 & 43 \\
10 & 0 & 1 & 0 & 3 & 2 & 6 & 5 & 10 & 8 & 17 & 14 & 25 & 22 & 35 & 30 & 48 & 43 & 64 & 58 \\
12 & 0 & 2 & 1 & 4 & 4 & 9 & 8 & 16 & 13 & 23 & 22 & 34 & 32 & 49 & 44 & 64 & 60 & 84 & 80 \\
14 & 0 & 2 & 1 & 5 & 5 & 12 & 11 & 19 & 17 & 29 & 28 & 44 & 42 & 60 & 56 & 79 & 77 & 106 & 102 \\
16 & 0 & 2 & 2 & 7 & 7 & 15 & 15 & 24 & 23 & 38 & 37 & 55 & 54 & 74 & 73 & 100 & 98 & 130 & 128 \\
18 & 0 & 4 & 3 & 9 & 10 & 19 & 20 & 32 & 31 & 47 & 48 & 68 & 69 & 94 & 92 & 122 & 123 & 158 & 159 \\
20 & 0 & 4 & 4 & 12 & 13 & 24 & 26 & 39 & 39 & 58 & 60 & 84 & 86 & 113 & 113 & 147 & 150 & 191 & 194 \\
32 & 1 & 12 & 18 & 37 & 46 & 71 & 82 & 111 & 121 & 160 & 175 & 221 & 238 & 290 & 306 & 370 & 391 & 465 & 489 \\
\hline
\end{tabular}

Table 2. $\operatorname{dim} J_{(k, j), 1}^{\text {cusp }}\left(\Gamma_{2}^{J}\right)$. (The values for $k \leq 6$ are conjectural for $j \geq 8$.) For odd $k$ we have $\operatorname{dim} J_{(k, j), 1}^{\text {cusp }}\left(\Gamma_{2}^{J}\right)=\operatorname{dim} J_{(k, j), 1}\left(\Gamma_{2}^{J}\right)$.

\begin{tabular}{|c|ccccccccc|}
\hline$j \backslash k$ & 4 & 6 & 8 & 10 & 12 & 14 & 16 & 18 & 20 \\
\hline 0 & 0 & 0 & 0 & 1 & 1 & 2 & 4 & 4 & 6 \\
2 & 0 & 0 & 1 & 1 & 2 & 4 & 6 & 8 & 13 \\
4 & 0 & 0 & 1 & 2 & 4 & 7 & 10 & 15 & 21 \\
6 & 0 & 0 & 2 & 4 & 7 & 11 & 17 & 23 & 32 \\
8 & 0 & 1 & 3 & 6 & 10 & 17 & 24 & 33 & 46 \\
10 & 0 & 1 & 4 & 8 & 14 & 22 & 32 & 44 & 60 \\
\hline
\end{tabular}

\begin{tabular}{|c|ccccccccc|}
\hline$j \backslash k$ & 4 & 6 & 8 & 10 & 12 & 14 & 16 & 18 & 20 \\
\hline 12 & 0 & 2 & 7 & 13 & 20 & 31 & 45 & 60 & 80 \\
14 & 0 & 3 & 9 & 16 & 26 & 40 & 56 & 75 & 101 \\
16 & 0 & 4 & 12 & 21 & 34 & 51 & 70 & 95 & 125 \\
18 & 1 & 6 & 16 & 28 & 43 & 64 & 89 & 117 & 153 \\
20 & 1 & 9 & 20 & 35 & 54 & 79 & 108 & 142 & 185 \\
32 & 7 & 32 & 65 & 105 & 154 & 214 & 283 & 363 & 457 \\
\hline
\end{tabular}

\section{Acknowledgements}

The author was partly supported by Grant-in-Aid A for Scientific Research No. 25247001, Japan Society of the Promotion of Science. 


\section{References}

[1] T. Arakawa, Vector-valued Siegel's modular forms of degree two and the associated Andrianov L-functions, Manuscripta Math. 44 (1983), 155-185. Zbl 0517.10024 MR 0709851

[2] J. Dulinski, A decomposition theorem for Jacobi forms, Math. Ann. 303 (1995), 473-498. Zbl 0882.11027 MR 1355001

[3] M. Eichler and D. Zagier, The theory of Jacobi forms. Progr. Math. 55, Birkhäuser Boston, Boston, MA, 1985. Zbl 0554.10018 MR 0781735

[4] E. Freitag, Ein Verschwindungssatz für automorphe Formen zur Siegelschen Modulgruppe, Math. Z. 165 (1979), 11-18. Zbl 0393.10027 MR 0521517

[5] _ Siegelsche Modulfunktionen, Grundlehren Math. Wiss. 254, Springer, Berlin, 1983. Zbl 0498.10016 MR 0871067

[6] S. Hayashida and T. Ibukiyama, Siegel modular forms of half integral weight and a lifting conjecture, J. Math. Kyoto Univ. 45 (2005), 489-530. Zbl 1122.11028 MR 2206360

[7] T. Ibukiyama, On Jacobi forms and Siegel modular forms of half integral weights, Comment. Math. Univ. St. Pauli 41 (1992), 109-124. Zbl 0787.11015 MR 3185225

[8] - On differential operators on automorphic forms and invariant pluri-harmonic polynomials, Comment. Math. Univ. St. Pauli 48 (1999), 103-118. Zbl 1007.11023 MR 1684769

[9] _ A conjecture on a Shimura type correspondence for Siegel modular forms, and Harder's conjecture on congruences, in: Modular forms on Schiermonnikoog, Cambridge Univ. Press, 2008, 107-144. Zbl 1259.11048 MR 2512360

[10] The Taylor expansion of Jacobi forms and applications to higher indices of degree two, Publ. RIMS Kyoto Univ. 48 (2012), 579-613. Zbl 1308.11051 MR 2973394

[11] Vector valued Siegel modular forms of symmetric tensor weight of small degrees, Comment. Math. Univ. St. Pauli 61 (2012), 51-75. Zbl 1287.11064 MR 3012313

[12] Conjectures of Shimura type and of Harder type revisited, Comment. Math. Univ. St. Pauli 63 (2014), 79-103. Zbl 06438539

[13] T. Ibukiyama and H. Kitayama, Dimension formulas of paramodular forms of squarefree level and comparison with inner twist, preprint, 2014, $59 \mathrm{pp}$.

[14] T. Ibukiyama and S. Wakatsuki, Siegel modular forms of small weight and the Witt operator, in: Quadratic forms - algebra, arithmetic, and geometry, Comtemp. Math. 493, Amer. Math. Soc., Providence, RI, 2009, 189-209. Zbl 1244.11049 MR 2537101

[15] S. Kimura, On vector valued Siegel modular forms of half integral weight and Jacobi forms, Master thesis, Osaka Univ., 2005, 82 pp. (in Japanese)

[16] I. Sataka, Surjectivité globale de l'opérateur $\Phi$, in Séminaire H. Cartan 1957/58, fonctions automorphes, exp. 16, École Normale Supérieure, 1958.

[17] N. P. Skoruppa, Über den Zusammenhang zwischen Jacobi-Formen und Modulformen halbganzen Gewichts, Bonner Math. Schriften 159, Univ. Bonn, 1985. Zbl 0564.10024 MR 0806354

[18] R. Tsushima, An explicit dimension formula for the spaces of generalized automorphic forms with respect to $\operatorname{Sp}(2, \mathbb{Z})$, Proc. Japan Acad. Ser. A Math. Sci. 59 (1983), 139-142. Zbl 0513.10025 MR 0711319

[19] On the dimension formula for the spaces of Jacobi forms of degree two, in Automorphic forms and L-functions (Kyoto, 1999), Sūrikaisekikenkyūsho Kōkyūroku 1103 (1999), 96-110. Zbl 0951.11515 MR 1746313

[20] Dimension formula for the spaces of Jacobi forms of degree two, preprint, 2010, 49 pp. 
[21] S. Wakatsuki, Dimension formulas for spaces of vector-valued Siegel cusp forms of degree two, J. Number Theory 132 (2012), 200-253. Zbl 1269.11051 MR 2843308

[22] C. Ziegler, Jacobi forms of higher degree, Abh. Math. Sem. Univ. Hamburg 59 (1989), 191-224. Zbl 0707.11035 MR 1049896 